TRANSACTIONS OF THE

AMERICAN MATHEMATICAL SOCIETY

Volume 364, Number 10, October 2012, Pages 5209-5242

S 0002-9947(2012)05493-6

Article electronically published on May 2, 2012

\title{
FAMILY INDEPENDENCE FOR TOPOLOGICAL AND MEASURABLE DYNAMICS
}

\author{
WEN HUANG, HANFENG LI, AND XIANGDONG YE
}

\begin{abstract}
For a family $\mathcal{F}$ (a collection of subsets of $\mathbb{Z}_{+}$), the notion of $\mathcal{F}$ independence is defined both for topological dynamics (t.d.s.) and measurable dynamics (m.d.s.). It is shown that there is no non-trivial \{syndetic\}independent m.d.s.; an m.d.s. is \{positive-density\}-independent if and only if it has completely positive entropy; and an m.d.s. is weakly mixing if and only if it is $\{$ IP $\}$-independent. For a t.d.s. it is proved that there is no non-trivial minimal \{syndetic $\}$-independent system; a t.d.s. is weakly mixing if and only if it is $\{\mathrm{IP}\}$-independent.

Moreover, a non-trivial proximal topological K system is constructed, and a topological proof of the fact that minimal topological $\mathrm{K}$ implies strong mixing is presented.
\end{abstract}

\section{INTRODUCTION}

By a topological dynamical system (t.d.s.) $(X, T)$ we mean a compact metrizable space $X$ together with a surjective continuous map $T$ from $X$ to itself. For a t.d.s. $(X, T)$ and non-empty open subsets $U$ and $V$ of $X$, let $N(U, V)=\left\{n \in \mathbb{Z}_{+}\right.$: $\left.U \cap T^{-n} V \neq \emptyset\right\}$, where $\mathbb{Z}_{+}$denotes the set of non-negative integers. It turns out that many recurrence properties of t.d.s. can be described using the return times sets $N(U, V)$; see [1, [12, [15, [29, [30]. For example, for a t.d.s. $(X, T)$ it is known that $T$ is (topologically) strongly mixing iff $N(U, V)$ is cofinite, $T$ is (topologically) weakly mixing iff $N(U, V)$ is thick 12 and $T$ is (topologically) mildly mixing iff $N(U, V)$ is an (IP - IP)* set [30, 21], for each pair of non-empty open subsets $U$ and $V$. Huang and Ye [30] showed that a minimal system $(X, T)$ is weakly mixing iff the lower Banach density of $N(U, V)$ is 1 and that $(X, T)$ is mildly mixing iff $N(U, V)$ is an $\mathrm{IP}^{*}$ set, for each pair of non-empty open sets $U$ and $V$.

By a measurable dynamical system (m.d.s.) we mean a quadruple $(X, \mathcal{B}, \mu, T)$, where $(X, \mathcal{B}, \mu)$ is a Lebesgue space (i.e., $X$ is a set, $\mathcal{B}$ is the $\sigma$-algebra of Borel subsets on $X$ for some Polish topology on $X$, and $\mu$ is a probability measure on $\mathcal{B}$ ) and $T: X \rightarrow X$ is measurable and measure-preserving; that is, $\mu(B)=\mu\left(T^{-1} B\right)$ for each $B \in \mathcal{B}$. For a t.d.s. $(X, T)$, there are always invariant Borel probability measures on $X$, and thus for each such measure $\mu,\left(X, \mathcal{B}_{X}, \mu, T\right)$, with $\mathcal{B}_{X}$ the Borel

Received by the editors December 14, 2009 and, in revised form, August 25, 2010.

2010 Mathematics Subject Classification. Primary 37B40, 37A35, 37B10, 37A05.

Key words and phrases. Independence, weak mixing, minimal, K.

The first author was partially supported by the NNSF of China (10911120388), the Fok Ying Tung Education Foundation, FANEDD (Grant 200520) and the Fundamental Research Funds for the Central Universities.

The second author was partially supported by NSF grant DMS-0701414.

The first and third authors were partially supported by grants from NNSF of China (10531010, 11071231) and 973 Project (2006CB805903). 
$\sigma$-algebra on $X$, is an m.d.s. For an m.d.s. $(X, \mathcal{B}, \mu, T)$, let $\mathcal{B}^{+}=\{B \in \mathcal{B}: \mu(B)>$ $0\}$ and $N(A, B)=\left\{n \in \mathbb{Z}_{+}: \mu\left(A \cap T^{-n} B\right)>0\right\}$ for $A, B \in \mathcal{B}^{+}$. It is known that $T$ is ergodic iff $N(A, B) \neq \emptyset$ iff $N(A, B)$ is syndetic, $T$ is weakly mixing iff the lower Banach density of $N(A, B)$ is 1 iff $N(A, B)$ is thick, and $T$ is mildly mixing iff $N(A, B)$ is an $\mathrm{IP}^{*}$ set iff $N(A, B)$ is an (IP - IP)* set for all $A, B \in \mathcal{B}^{+}$iff for each IP set $F$ and $A \in \mathcal{B}^{+}, \mu\left(\bigcup_{n \in F} T^{-n} A\right)=1$. Finally, it is known that $T$ is intermixing iff $N(A, B)$ is cofinite for all $A, B \in \mathcal{B}^{+}$; see [37, 38] and the references therein.

In ergodic theory there exists a rich and powerful entropy theory. The analogous notion of topological entropy was introduced soon after the measure theoretical one and was widely studied and applied. Notwithstanding, the level of development of topological entropy theory lagged behind. In recent years however this situation has rapidly changed. A turning point occurred with F. Blanchard's pioneering papers [4], 5] in the 1990s.

In recent years a local entropy theory has been developed; see 22 for a survey. More precisely, in [4 Blanchard introduced the notions of completely positive entropy (c.p.e.) and uniformly positive entropy (u.p.e.) as topological analogues of the $K$-property in ergodic theory. In [5] he defined the notion of entropy pairs and used it to show that a u.p.e. system is disjoint from all minimal zero entropy systems. The notion of entropy pairs can also be used to show the existence of the maximal zero entropy factor for any t.d.s., namely the topological Pinsker factor 8. Blanchard et al. 7] also introduced the notion of entropy pairs for an invariant Borel probability measure. Glasner and Weiss [19] introduced the notion of entropy tuples. In order to gain a better understanding of the topological version of a Ksystem, Huang and Ye 32 introduced the notion of entropy tuples for an invariant Borel probability measure. They showed that if $(X, T)$ is a t.d.s. and $k \geq 2$, then a non-diagonal tuple $\left(x_{1}, \ldots, x_{k}\right)$ in $X^{k}$ is an entropy tuple iff for every choice of neighborhoods $U_{i}$ of $x_{i}$ there is a subset $F$ of $\mathbb{Z}_{+}$with positive density such that $\bigcap_{i \in F} T^{-i} U_{s(i)} \neq \emptyset$ for each $s \in\{1, \ldots, k\}^{F}$. We mention that at the same time a theory of sequence entropy tuples and tame systems were developed [16, 26, 17. It is Kerr and $\mathrm{Li}$ who captured the idea behind the results on entropy tuples, sequence entropy tuples and tame systems and who treated them systematically using a notion called independence in [35, 36], which first appeared in Rosenthal's proof of his groundbreaking $\ell_{1}$ theorem [45, 46].

Let $(X, T)$ be a t.d.s. For a tuple $\mathbf{A}=\left(A_{1}, \ldots, A_{k}\right)$ of subsets of $X$, we say a subset $F \subseteq \mathbb{Z}_{+}$is an independence set for $\mathbf{A}$ if for any non-empty finite subset $J \subseteq F$, we have

$$
\bigcap_{j \in J} T^{-j} A_{s(j)} \neq \emptyset
$$

for any $s \in\{1, \ldots, k\}^{J}$. We call a tuple $\mathbf{x}=\left(x_{1}, \ldots, x_{k}\right) \in X^{k}(1)$ an IE-tuple if for every product neighborhood $U_{1} \times \cdots \times U_{k}$ of $\mathbf{x}$ the tuple $\left(U_{1}, \ldots, U_{k}\right)$ has an independence set of positive density; (2) an IT-tuple if for every product neighborhood $U_{1} \times \cdots \times U_{k}$ of $\mathbf{x}$ the tuple $\left(U_{1}, \ldots, U_{k}\right)$ has an infinite independence set; (3) an $I N$-tuple if for every product neighborhood $U_{1} \times \cdots \times U_{k}$ of $\mathbf{x}$ the tuple $\left(U_{1}, \ldots, U_{k}\right)$ has arbitrarily long finite independence sets. Kerr and Li [35] showed that (1) entropy tuples are exactly non-diagonal IE-tuples; (2) sequence entropy tuples are exactly non-diagonal IN-tuples, and in particular a t.d.s. $(X, T)$ is null iff it has no 
non-diagonal IN-pairs; (3) a t.d.s. $(X, T)$ is tame iff it has no non-diagonal IT-pairs. For similar results concerning m.d.s. see [36].

Thus the notion of independence is very useful to describe dynamical properties. A family is a collection of subsets of the set of non-negative integers satisfying suitable hereditary property. For a family $\mathcal{F}$, the notion of $\mathcal{F}$-independence can be defined both for topological dynamics (t.d.s.) and measurable dynamics (m.d.s.). For instance, in the topological case, we say that $(X, T)$ is $\mathcal{F}$-independent if every finite tuple of subsets of $X$ with non-empty interiors has an independence set in $\mathcal{F}$. So a natural question is: for a given family $\mathcal{F}$, which dynamical property is equivalent to $\mathcal{F}$-independence? In this paper we try to answer this question.

It is shown that there is no non-trivial \{syndetic\}-independent m.d.s.; an m.d.s. is \{positive-density\}-independent iff it has completely positive entropy; and an m.d.s. is weakly mixing iff it is $\{$ infinite $\}$-independent iff it is $\{\mathrm{IP}\}$-independent. For a t.d.s. it is proved that there is no non-trivial minimal \{syndetic $\}$-independent system; a t.d.s. is weakly mixing iff it is $\{$ infinite $\}$-independent iff it is $\{$ IP $\}$ independent.

Moreover, a non-trivial proximal topological K-system (see Definition 5.3 below) is constructed, and a topological proof (using independence) of the fact that minimal topological $\mathrm{K}$ implies strong mixing is presented. In a forthcoming paper [27] we will deal with the problem of how to localize the notion of $\mathcal{F}$-independence.

In 5] Blanchard raised the question of whether there exists any non-trivial minimal uniformly positive entropy (equivalently, \{positive-density $\}$-independent of order 2 in our terminology) t.d.s. This was answered affirmatively by Glasner and Weiss in 18. Later Huang and Ye showed there are non-trivial minimal \{positivedensity\}-independent t.d.s. [32]. However, the constructions in [18] and [32] are based on showing that any minimal topological model of a K-system is such an example and then using the Jewett-Krieger theorem to obtain such a topological model. So far there is no explicit topological construction of such examples. Since the family of syndetic sets is just slightly smaller than the family of positive upper Banach density sets, our result of the non-existence of non-trivial minimal \{syndetic $\}$-independent t.d.s. explains why it is so difficult to construct examples for Blanchard's question.

The paper is organized as follows. In Section 2, we investigate the relationship between a given family $\mathcal{F}$ and the associated block family $b \mathcal{F}$. In Section 3 , the basic properties of $\mathcal{F}$-independence for a t.d.s. are discussed. Particularly, we show that $\mathcal{F}$ and $b \mathcal{F}$ define the same notion of independence. In Section 4 , the basic properties of $\mathcal{F}$-independence for an m.d.s. are discussed. In Section 5 , we investigate classes of $\mathcal{F}$-independent systems for t.d.s. and show that there is no non-trivial minimal \{syndetic\}-independent t.d.s. Moreover, a non-trivial proximal topological K-system is constructed. In Section 6 , we investigate classes of $\mathcal{F}$ independent systems for m.d.s. and show that an m.d.s. is \{positive-density\}independent iff it has completely positive entropy. We also show that there is no non-trivial \{syndetic\}-independent m.d.s. In Section 7, we give a topological proof of the fact that minimal topological $\mathrm{K}$ implies strong mixing. An interesting combinatorial result, which is needed for the proof of non-existence of non-trivial minimal \{syndetic\}-independent t.d.s., is established in the Appendix.

Throughout this paper, we use $\mathbb{Z}_{+}$and $\mathbb{N}$ to denote the sets of non-negative integers and positive integers, respectively. For a subset $F$ of $\mathbb{Z}$ and $m \in \mathbb{Z}$ we 
denote $\{j+m: j \in F\}$ by $F+m$. For a subshift $X$ of $\{0,1, \ldots, k\}^{\mathbb{Z}_{+}}$or $\{0,1, \ldots, k\}^{\mathbb{Z}}$ and $a \in\{0,1, \ldots, k\}^{\{1, \ldots, m\}}$ for some $m \in \mathbb{N}$, we denote $\{x \in X:(x(0), x(1), \ldots$, $x(m-1))=a\}$ by $[a]_{X}$. For a t.d.s. $(X, T)$ and subsets $U, V \subseteq X$, we denote by $N(U, V)$ the set $\left\{n \in \mathbb{Z}_{+}: U \bigcap T^{-n} V \neq \emptyset\right\}$; for $x \in X$ we shall write $N(x, U)$ for $N(\{x\}, U)$. For an m.d.s. $(X, \mathcal{B}, \mu, T)$ and $A, B \in \mathcal{B}$, we denote by $N(A, B)$ the set $\left\{n \in \mathbb{Z}_{+}: \mu\left(A \cap T^{-n} B\right)>0\right\}$.

\section{Preliminary}

The idea of using families to describe dynamical properties goes back at least to Gottschalk and Hedlund [23. It was developed further by Furstenberg [12, 13]. For a systematic study and recent results, see [1, 15, 29, 30].

Let us recall some notation related to a family (for details see [1). Let $\mathcal{P}=$ $\mathcal{P}\left(\mathbb{Z}_{+}\right)$be the collection of all subsets of $\mathbb{Z}_{+}$. A subset $\mathcal{F}$ of $\mathcal{P}$ is a family if it is hereditary upward. That is, $F_{1} \subseteq F_{2}$ and $F_{1} \in \mathcal{F}$ imply $F_{2} \in \mathcal{F}$. A family $\mathcal{F}$ is proper if it is a proper subset of $\mathcal{P}$, i.e. neither empty nor all of $\mathcal{P}$. It is easy to see that $\mathcal{F}$ is proper if and only if $\mathbb{Z}_{+} \in \mathcal{F}$ and $\emptyset \notin \mathcal{F}$. Any subset $\mathcal{A}$ of $\mathcal{P}$ generates a family $[\mathcal{A}]=\{F \in \mathcal{P}: F \supseteq A$ for some $A \in \mathcal{A}\}$. If a proper family $\mathcal{F}$ is closed under taking finite intersection, then $\mathcal{F}$ is called a filter. For a family $\mathcal{F}$, the dual family is

$$
\mathcal{F}^{*}=\left\{F \in \mathcal{P}: \mathbb{Z}_{+} \backslash F \notin \mathcal{F}\right\}=\left\{F \in \mathcal{P}: F \cap F^{\prime} \neq \emptyset \text { for all } F^{\prime} \in \mathcal{F}\right\} .
$$

$\mathcal{F}^{*}$ is a family, proper if $\mathcal{F}$ is proper. Clearly,

$$
\left(\mathcal{F}^{*}\right)^{*}=\mathcal{F} \text { and } \mathcal{F}_{1} \subseteq \mathcal{F}_{2} \Longrightarrow \mathcal{F}_{2}^{*} \subseteq \mathcal{F}_{1}^{*}
$$

There is an important property being well studied: the Ramsey property. We say that a family $\mathcal{F}$ has the Ramsey property if whenever $F_{1} \cup F_{2} \in \mathcal{F}$, one has either $F_{1} \in \mathcal{F}$ or $F_{2} \in \mathcal{F}$. One can show that a proper family $\mathcal{F}$ has the Ramsey property if and only if $\mathcal{F}^{*}$ is a filter [1, page 26].

Denote by $\mathcal{F}_{\text {inf }}$ the family of all infinite subsets of $\mathbb{Z}_{+}$and by $\mathcal{F}_{\mathrm{c}}$ the dual family $\mathcal{F}_{\text {inf }}^{*}$. Note that $\mathcal{F}_{\mathrm{c}}$ is the collection of all cofinite subsets of $\mathbb{Z}_{+}$. All the families considered in this paper are assumed to be proper and contained in $\mathcal{F}_{\text {inf }}$.

Let $F$ be a subset of $\mathbb{Z}_{+}$. The lower density and upper density of $F$ are defined by

$\underline{d}(F)=\liminf _{n \rightarrow+\infty} \frac{1}{n}|F \cap\{0,1, \ldots, n-1\}|$ and $\bar{d}(F)=\limsup _{n \rightarrow+\infty} \frac{1}{n}|F \cap\{0,1, \ldots, n-1\}|$.

If $\underline{d}(F)=\bar{d}(F)=d(F)$, we then say that the density of $F$ is $d(F)$. The upper Banach density of $F$ is defined by

$$
B D^{*}(F)=\limsup _{|I| \rightarrow+\infty} \frac{|S \cap I|}{|I|}
$$

where $I$ is taken over all non-empty finite intervals of $\mathbb{Z}_{+}$.

We denote by $\mathcal{F}_{\text {pd }}$ the family generated by sets with positive density, by $\mathcal{F}_{\text {pud }}$ the family of sets with positive upper density, and by $\mathcal{F}_{\text {pubd }}$ the family of sets with positive upper Banach density.

Note that a subset $F$ of $\mathbb{Z}_{+}$is said to be thick if for any $n \in \mathbb{N}$ there exists some $m \in \mathbb{Z}_{+}$such that $\{m, m+1, \ldots, m+n\} \subseteq F$. An infinite subset $F=\left\{s_{1}<s_{2}<\right.$ $\cdots\}$ of $\mathbb{Z}_{+}$is said to be syndetic if $\left\{s_{n+1}-s_{n}: n \in \mathbb{N}\right\}$ is bounded. A subset of $\mathbb{Z}_{+}$ is called piecewise syndetic if it is the intersection of a thick set and a syndetic set. 
We denote by $\mathcal{F}_{\mathrm{t}}, \mathcal{F}_{\mathrm{s}}$ and $\mathcal{F}_{\mathrm{ps}}$ the families of thick sets, syndetic sets and piecewise syndetic sets, respectively.

A subset $F$ of $\mathbb{Z}_{+}$is called a central set if there exists a t.d.s. $(X, T)$, a point $x \in X$, a minimal point $y \in X$ which is proximal to $x$ and a neighborhood $U_{y}$ of $y$ such that $F \supseteq N\left(x, U_{y}\right)$ [13. Section 8.3]. Here $y$ is proximal to $x$ means that for a compatible metric $d$ of $X$, one has $\inf _{n \in \mathbb{Z}_{+}} d\left(T^{n} x, T^{n} y\right)=0$. We denote by $\mathcal{F}_{\text {cen }}$ the family of all central sets.

A subset $F$ of $\mathbb{Z}_{+}$is called an IP-set if there exists a sequence $\left\{a_{n}\right\}_{n \in \mathbb{N}}$ in $\mathbb{N}$ such that $F$ consists of $a_{n_{1}}+a_{n_{2}}+\cdots+a_{n_{k}}$ for all $k \in \mathbb{N}$ and $n_{1}<n_{2}<\cdots<n_{k}$. We denote by $\mathcal{F}_{\text {ip }}$ the family generated by all IP-sets.

Definition 2.1. Let $\mathcal{F}$ be a family. The block family of $\mathcal{F}$, denoted by $b \mathcal{F}$, is the family consisting of sets $S \subseteq \mathbb{Z}_{+}$for which there exists some $F \in \mathcal{F}$ such that for every finite subset $W$ of $F$ one has $m+W \subseteq S$ for some $m \in \mathbb{Z}$.

Clearly $\mathcal{F} \subseteq b \mathcal{F}$ and $b(b \mathcal{F})=b \mathcal{F}$. It is also clear that $b \mathcal{F}_{\text {inf }}=\mathcal{F}_{\text {inf }}$ and $b \mathcal{F}_{\mathrm{c}}=\mathcal{F}_{\mathrm{t}}$.

Example 2.2. It is clear that $b \mathcal{F}_{\text {pd }} \subseteq b \mathcal{F}_{\text {pud }} \subseteq \mathcal{F}_{\text {pubd. }}$. It is a result of Ellis that $\mathcal{F}_{\text {pubd }} \subseteq b \mathcal{F}_{\text {pd }}[13$. Theorem 3.20] (one can also give a topological proof for this, using an argument similar to that in the proof of Lemma 4.5). Thus one has $b \mathcal{F}_{\text {pd }}=b \mathcal{F}_{\text {pud }}=\mathcal{F}_{\text {pubd }}$.

Example 2.3. It is clear that $b \mathcal{F}_{\mathrm{s}} \subseteq \mathcal{F}_{\mathrm{ps}}$. Let $S_{1} \in \mathcal{F}_{\mathrm{t}}$ and $S_{2} \in \mathcal{F}_{\mathrm{s}}$. Then for each $n \in \mathbb{N}$ we can find some $a_{n} \in \mathbb{Z}_{+}$with $\left[a_{n}, a_{n}+n\right] \subseteq S_{1}$. Some subsequence of the sequence $\left\{1_{\left(\left[a_{n}, a_{n}+n\right] \cap S_{2}\right)-a_{n}}\right\}_{n \in \mathbb{N}}$ converges in $\{0,1\}^{\mathbb{Z}_{+}}$to $1_{F}$ for some subset $F$ of $\mathbb{Z}_{+}$. It is easy to see that $F$ is syndetic and that for every finite subset $W$ of $F$ one has $m+W \subseteq S_{1} \cap S_{2}$ for some $m \in \mathbb{Z}_{+}$. Therefore $b \mathcal{F}_{\mathrm{s}} \supseteq \mathcal{F}_{\mathrm{ps}}$, and hence $b \mathcal{F}_{\mathrm{s}}=\mathcal{F}_{\mathrm{ps}}$.

Example 2.4. It is clear that $\mathcal{F}_{\text {cen }} \subseteq \mathcal{F}_{\mathrm{ps}}$ and hence $b \mathcal{F}_{\text {cen }} \subseteq b \mathcal{F}_{\mathrm{ps}}=\mathcal{F}_{\mathrm{ps}}$. Let $S \in$ $\mathcal{F}_{\text {ps }}$. Denote by $X$ the smallest closed shift-invariant subset of $\{0,1\}^{\mathbb{Z}}$ containing $1_{S}$. Note that $S=N\left(1_{S},[1]_{X}\right)$. By [9, Theorem 6] there is a minimal point $x$ of $X$ contained in $[1]_{X}$. Say $x=1_{S^{\prime}}$. Set $F=S^{\prime} \cap \mathbb{Z}_{+}$. Then $F=N\left(x,[1]_{X}\right)$ is central. Since $x$ is in $X$, it is easy to see that for every finite subset $W$ of $F$ one has $m+W \subseteq S$ for some $m \in \mathbb{Z}$. This means that $S \in b \mathcal{F}_{\text {cen }}$. Therefore $b \mathcal{F}_{\text {cen }} \supseteq \mathcal{F}_{\mathrm{ps}}$, and hence $b \mathcal{F}_{\text {cen }}=\mathcal{F}_{\text {ps }}$.

The following result shows the relation between the block family and the broken family introduced in [9, Defintion 2].

Proposition 2.5. Let $\mathcal{F}$ be a family. Let $S \subseteq \mathbb{Z}_{+}$. Then $S \in b \mathcal{F}$ if and only if there exist an $F=\left\{p_{1}<p_{2}<\cdots\right\} \in \mathcal{F}$ and a (not necessarily strictly) increasing sequence $\left\{b_{j}\right\}_{j=1}^{\infty}$ of integers such that $S \supseteq \bigcup_{j=1}^{\infty}\left\{b_{j}+\left\{p_{1}, p_{2}, \ldots, p_{j}\right\}\right\}$.

Proof. The "if" part is trivial.

Suppose that $S \in b \mathcal{F}$. Let $F=\left\{p_{1}<p_{2}<\cdots\right\} \in \mathcal{F}$ witnessing this. Then for each $j \in \mathbb{N}$ we find some $b_{j} \in \mathbb{Z}$ with $b_{j}+\left\{p_{1}, \ldots, p_{j}\right\} \subseteq S$. Note that $b_{j}+p_{1} \geq 0$ for every $j \in \mathbb{N}$. Thus we can find an increasing subsequence $\left\{b_{j_{k}}\right\}_{k=1}^{\infty}$ of $\left\{b_{j}\right\}_{j=1}^{\infty}$. Then for each $k \in \mathbb{N}$ we have $b_{j_{k}}+\left\{p_{1}, \ldots, p_{k}\right\} \subseteq b_{j_{k}}+\left\{p_{1}, \ldots, p_{j_{k}}\right\} \subseteq S$. Thus $S \supseteq \bigcup_{k=1}^{\infty}\left\{b_{j_{k}}+\left\{p_{1}, p_{2}, \ldots, p_{k}\right\}\right\}$. This proves the "only if" part.

The next result follows from Proposition 3.7 and Lemma 3.9, which we shall prove in the next section. 
Proposition 2.6. If $\mathcal{F}$ has the Ramsey property, then so does $b \mathcal{F}$.

We remark that if $b \mathcal{F}$ has the Ramsey property, it is not necessarily true that $\mathcal{F}$ has the Ramsey property. For example, $\mathcal{F}_{\text {pud }}$ and $\mathcal{F}_{\text {pubd }}$ have the Ramsey property, while $\mathcal{F}_{\text {pd }}$ does not.

For the reader's convenience we make the following table. All the definitions of the families can be found in this section except $\mathcal{F}_{\text {ss }}$ and $\mathcal{F}_{\text {rs }}$, which can be found in Section 7 and Section 3 respectively.

TABLE 1. Notions for families

\begin{tabular}{|c|l|l|l|}
\hline $\mathcal{F}_{\text {ss }}$ & \multicolumn{1}{|c|}{$\mathcal{F}_{\text {inf }}$} & \multicolumn{1}{c|}{$\mathcal{F}_{\text {pubd }}$} & $\mathcal{F}_{\text {ps }}$ \\
\hline Section 7 & $\begin{array}{l}\text { all infinite } \\
\text { sets }\end{array}$ & $\begin{array}{l}\text { all positive } \\
\text { upper Banach } \\
\text { density sets }\end{array}$ & $\begin{array}{l}\text { all piecewise syn- } \\
\text { detic sets }\end{array}$ \\
\hline $\mathcal{F}_{\mathrm{s}}$ & $\mathcal{F}_{\text {pd }}$ & $\mathcal{F}_{\text {rs }}$ & $\mathcal{F}_{\mathrm{c}}$ \\
\hline all syndetic sets & $\begin{array}{l}\text { generated by } \\
\text { all positive } \\
\text { density sets }\end{array}$ & $\begin{array}{l}\text { generated by } \\
\left\{n \mathbb{Z}_{+}: n \in \mathbb{N}\right\}\end{array}$ & all cofinite sets \\
\hline $\mathcal{F}_{\text {ip }}$ & $\mathcal{F}_{\text {cen }}$ & $\mathcal{F}_{\mathrm{t}}$ & $\mathcal{F}_{\text {pud }}$ \\
\hline $\begin{array}{l}\text { generated by all } \\
\text { IP-sets }\end{array}$ & $\begin{array}{l}\text { all central } \\
\text { sets }\end{array}$ & all thick sets & $\begin{array}{l}\text { all positive upper } \\
\text { density sets }\end{array}$ \\
\hline
\end{tabular}

\section{IndePendence: TOPOlOGiCAl CASE}

In this section, for a given family $\mathcal{F}$, we define $\mathcal{F}$-independence for t.d.s. and discuss 1-independence for various families. First recall the notion of independence set introduced in [35, Definition 2.1].

Definition 3.1. Let $(X, T)$ be a t.d.s. For a tuple $\mathbf{A}=\left(A_{1}, \ldots, A_{k}\right)$ of subsets of $X$, we say that a subset $F \subseteq \mathbb{Z}_{+}$is an independence set for $\mathbf{A}$ if for any non-empty finite subset $J \subseteq F$, we have

$$
\bigcap_{j \in J} T^{-j} A_{s(j)} \neq \emptyset
$$

for any $s \in\{1, \ldots, k\}^{J}$.

We shall denote the collection of all independence sets for $\mathbf{A}$ by $\operatorname{Ind}\left(A_{1}, \ldots, A_{k}\right)$ or Ind $\mathbf{A}$. The basic properties of independence sets are listed below.

Lemma 3.2. The following hold:

(1) If $F \in \operatorname{Ind}\left(A_{1}, \ldots, A_{k}\right)$ and $F_{1} \subseteq F$, then $F_{1} \in \operatorname{Ind}\left(A_{1}, \ldots, A_{k}\right)$.

(2) $F=\left\{a_{1}, a_{2}, \ldots\right\}$ is in $\operatorname{Ind}\left(A_{1}, \ldots, A_{k}\right)$ if and only if $\left\{a_{1}, \ldots, a_{n}\right\}$ is in $\operatorname{Ind}\left(A_{1}, \ldots, A_{k}\right)$ for each $n \in \mathbb{N}$.

(3) If $m \in \mathbb{Z}$ and $F, m+F \subseteq \mathbb{Z}_{+}$, then $F$ is in $\operatorname{Ind}\left(A_{1}, \ldots, A_{k}\right)$ if and only if $m+F$ is as well.

(4) Let $F \subseteq \mathbb{Z}_{+}$and $X$ be the subshift of $\{0,1\}^{\mathbb{Z}}$ generated by $\left\{1_{E}: E \subseteq F\right\}$. Then $F \in \operatorname{Ind}\left([0]_{X},[1]_{X}\right)$. 
Definition 3.3. Let $\mathcal{F}$ be a family. We say that $\mathcal{F}$ has the dynamical Ramsey property if, for any t.d.s. $(X, T)$, any $k \in \mathbb{N}$ and closed subsets $A_{1}, A_{2}, \ldots, A_{k}, A_{1,1}, A_{1,2}$ of $X$ with $A_{1}=A_{1,1} \cup A_{1,2}$, whenever $\operatorname{Ind}\left(A_{1}, A_{2}, \ldots, A_{k}\right) \cap \mathcal{F} \neq \emptyset$, one has either $\operatorname{Ind}\left(A_{1,1}, A_{2}, \ldots, A_{k}\right) \cap \mathcal{F} \neq \emptyset$ or $\operatorname{Ind}\left(A_{1,2}, A_{2}, \ldots, A_{k}\right) \cap \mathcal{F} \neq \emptyset$.

It was shown in [35. Lemmas 3.8 and 6.3] that the families $\mathcal{F}_{\text {pd }}$ and $\mathcal{F}_{\text {inf }}$ have the dynamical Ramsey property.

Similar to the definition of u.p.e. of order $n$ (see [32]), we have

Definition 3.4. Let $\mathcal{F}$ be a family, $k \in \mathbb{N}$ and $(X, T)$ be a t.d.s. A tuple $\left(x_{1}, \ldots, x_{k}\right) \in X^{k}$ is called an $\mathcal{F}$-independent tuple if for any neighborhoods $U_{1}, \ldots$, $U_{k}$ of $x_{1}, \ldots, x_{k}$, respectively, one has $\operatorname{Ind}\left(U_{1}, \ldots, U_{k}\right) \cap \mathcal{F} \neq \emptyset$. A t.d.s. is said to be $\mathcal{F}$-independent of order $k$ if for each tuple of non-empty open subsets $U_{1}, \ldots, U_{k}$, $\operatorname{Ind}\left(U_{1}, \ldots, U_{k}\right) \cap \mathcal{F} \neq \emptyset$, and a t.d.s. is said to be $\mathcal{F}$-independent if it is $\mathcal{F}$ independent of order $k$ for each $k \in \mathbb{N}$.

Standard arguments as in [5] show the following:

Proposition 3.5. Let $\mathcal{F}$ be a family with the dynamical Ramsey property, and let $(X, T)$ be a t.d.s. The following are true:

(1) If $\mathbf{A}=\left(A_{1}, \ldots, A_{k}\right)$ is a tuple of closed subsets of $X$ with $\operatorname{Ind} \mathbf{A} \cap \mathcal{F} \neq \emptyset$, then there exists $x_{j} \in A_{j}$ for each $1 \leq j \leq k$ such that $\left(x_{1}, \ldots, x_{k}\right)$ is an $\mathcal{F}$-independent tuple.

(2) Let $k \in \mathbb{N}$. Then the set of $\mathcal{F}$-independent $k$-tuples of $X$ is a closed $T \times$ $\cdots \times T$-invariant subset of $X^{k}$.

(3) Let $(Y, S)$ be a t.d.s. and $\pi: X \rightarrow Y$ be a factor map, i.e., $\pi$ is continuous surjective and equivariant. Let $k \in \mathbb{N}$. Then $\pi \times \cdots \times \pi$ maps the set of $\mathcal{F}$-independent $k$-tuples of $X$ onto the set of $\mathcal{F}$-independent $k$-tuples of $Y$.

Recall that two t.d.s. $(X, T)$ and $(Y, S)$ are said to be disjoint [12] if $X \times Y$ is the only closed subset $Z$ of $X \times Y$ satisfying $(T \times S)(Z)=Z$ and projecting surjectively to $X$ and $Y$ under the natural projections $X \times Y \rightarrow X$ and $X \times Y \rightarrow Y$, respectively. Following the arguments in the proofs of [5, Proposition 6] and [8, Theorem 2.1], we have

Theorem 3.6. Let $\mathcal{F}$ be a family with the dynamical Ramsey property. The following are true:

(1) Each t.d.s. which is $\mathcal{F}$-independent of order 2 is disjoint from every minimal system without non-diagonal $\mathcal{F}$-independent pairs.

(2) Each t.d.s. admits a maximal factor with no non-diagonal $\mathcal{F}$-independent pairs.

Different families might lead to the same notion of independence. In fact, it follows from Lemma $3.2(2)(3)$ that $\operatorname{Ind}\left(A_{1}, \ldots, A_{k}\right) \cap \mathcal{F} \neq \emptyset$ if and only if $\operatorname{Ind}\left(A_{1}, \ldots, A_{k}\right) \cap b \mathcal{F} \neq \emptyset$. Thus we have:

Proposition 3.7. Let $\mathcal{F}$ be a family. Then:

(1) The families $\mathcal{F}$ and $b \mathcal{F}$ define the same notion of independence.

(2) $\mathcal{F}$ has the dynamical Ramsey property if and only if $b \mathcal{F}$ does.

Theorem 3.8. Let $\mathcal{F}_{1}, \mathcal{F}_{2}$ be two families having the dynamical Ramsey property. Then $\mathcal{F}_{1}$-independent pairs are exactly the same as $\mathcal{F}_{2}$-independent pairs if and only if $b \mathcal{F}_{1}=b \mathcal{F}_{2}$. 
Proof. The "if" part follows from Proposition 3.7.

Now assume that each $\mathcal{F}_{1}$-independent pair is an $\mathcal{F}_{2}$-independent pair. We are going to show that $b \mathcal{F}_{1} \subseteq b \mathcal{F}_{2}$.

Let $F \in \mathcal{F}_{1}$. Denote by $X$ the smallest closed shift-invariant subset of $\{0,1\}^{\mathbb{Z}}$ containing $\left\{1_{E}: E \subseteq F\right\}$. Then $F \in \operatorname{Ind}\left([0]_{X},[1]_{X}\right)$ and

$$
X=\overline{\left\{T^{i} 1_{E}: i \in \mathbb{Z}, E \subseteq F\right\}}
$$

where $T$ denotes the shift. Since $\mathcal{F}_{1}$ has the dynamical Ramsey property, there exists $(x, y) \in[0]_{X} \times[1]_{X}$ which is $\mathcal{F}_{1}$-independent. As each $\mathcal{F}_{1}$-independent pair is an $\mathcal{F}_{2}$-independent pair, we get that $\operatorname{Ind}\left([0]_{X},[1]_{X}\right) \cap \mathcal{F}_{2} \neq \emptyset$. Let $F^{\prime} \in \operatorname{Ind}\left([0]_{X},[1]_{X}\right) \cap$ $\mathcal{F}_{2}$. For any finite subset $W$ of $F^{\prime}$, there exists $x_{W} \in \bigcap_{k \in W} T^{-k}\left([1]_{X}\right)$. Then $x_{W}(k)=1$ for every $k \in W$. Since $x_{W} \in X$, it follows that there exists some $m \in \mathbb{Z}$ with $m+W \subseteq F$. Therefore $F \in b \mathcal{F}_{2}$. Thus $\mathcal{F}_{1} \subseteq b \mathcal{F}_{2}$, and hence $b \mathcal{F}_{1} \subseteq b\left(b \mathcal{F}_{2}\right)=b \mathcal{F}_{2}$. This proves the "only if" part.

From Theorem 3.8 one sees that if a family $b \mathcal{F}$ has the dynamical Ramsey property, then among the families which have the dynamical Ramsey property and define the same independence as $\mathcal{F}$ does, $b \mathcal{F}$ is the largest one.

Lemma 3.9. Let $\mathcal{F}$ be a family. If $\mathcal{F}$ has the Ramsey property, then for any t.d.s. $(X, T)$ and closed subsets $Y, Y_{1}, Y_{2}$ of $X$ with $Y=Y_{1} \cup Y_{2}$ and $\operatorname{Ind}(Y) \cap \mathcal{F} \neq \emptyset$, one has either $\operatorname{Ind}\left(Y_{1}\right) \cap \mathcal{F} \neq \emptyset$ or $\operatorname{Ind}\left(Y_{2}\right) \cap \mathcal{F} \neq \emptyset$. The converse holds if furthermore $\mathcal{F}=b \mathcal{F}$.

Proof. Suppose that $\mathcal{F}$ has the Ramsey property. Consider a t.d.s. $(X, T)$ and closed subsets $Y, Y_{1}, Y_{2}$ of $X$ with $Y=Y_{1} \cup Y_{2}$ and $\operatorname{Ind}(Y) \cap \mathcal{F} \neq \emptyset$. Take $F \in$ $\operatorname{Ind}(Y) \cap \mathcal{F}$. Then $\bigcap_{n \in F} T^{-n}(Y) \neq \emptyset$. Say $x \in \bigcap_{n \in F} T^{-n} Y$. Set $F_{j}=\{n \in F$ : $\left.T^{n} x \in Y_{j}\right\}$ for $j=1,2$. Then $F=F_{1} \cup F_{2}$, and hence either $F_{1} \in \mathcal{F}$ or $F_{2} \in \mathcal{F}$. Thus either $\operatorname{Ind}\left(Y_{1}\right) \cap \mathcal{F} \neq \emptyset$ or $\operatorname{Ind}\left(Y_{2}\right) \cap \mathcal{F} \neq \emptyset$.

Now suppose that $\mathcal{F}=b \mathcal{F}$, and for any t.d.s. $(X, T)$ and closed subsets $Y, Y_{1}, Y_{2}$ of $X$ with $Y=Y_{1} \cup Y_{2}$ and $\operatorname{Ind}(Y) \cap \mathcal{F} \neq \emptyset$, one has either $\operatorname{Ind}\left(Y_{1}\right) \cap \mathcal{F} \neq \emptyset$ or $\operatorname{Ind}\left(Y_{2}\right) \cap \mathcal{F} \neq \emptyset$. Let $F \in \mathcal{F}$ and $F=F_{1} \cup F_{2}$ with $F_{1} \cap F_{2}=\emptyset$. Denote by $X$ the smallest closed shift-invariant subset of $\{0,1,2\}^{\mathbb{Z}}$ containing $1_{F_{1}}+2 \cdot 1_{F_{2}}$. Then $F \in \operatorname{Ind}\left([1]_{X} \cup[2]_{X}\right)$. By assumption we have either $\operatorname{Ind}\left([1]_{X}\right) \cap \mathcal{F} \neq \emptyset$ or $\operatorname{Ind}\left([2]_{X}\right) \cap \mathcal{F} \neq \emptyset$. Without loss of generality let us assume that $\operatorname{Ind}\left([1]_{X}\right) \cap \mathcal{F} \neq \emptyset$. Say $F^{\prime} \in \operatorname{Ind}\left([1]_{X}\right) \cap \mathcal{F}$. Since $X$ is the orbit closure of $1_{F_{1}}+2 \cdot 1_{F_{2}}$, it follows that for any finite subset $W$ of $F^{\prime}$ there exists some $m \in \mathbb{Z}$ with $m+W \subseteq F_{1}$. Thus $F_{1} \in b \mathcal{F}=\mathcal{F}$. Therefore $\mathcal{F}$ has the Ramsey property.

From Proposition 3.7 and Lemma 3.9 we get:

Proposition 3.10. Let $\mathcal{F}$ be a family. If $\mathcal{F}$ has the dynamical Ramsey property, then $b \mathcal{F}$ has the Ramsey property.

We remark that if $\mathcal{F}$ has the dynamical Ramsey property, it is not necessarily true that $\mathcal{F}$ has the Ramsey property. For example, $\mathcal{F}_{\mathrm{pd}}$ has the dynamical Ramsey property, but not the Ramsey property.

It is easy to see that $\mathcal{F}_{\mathrm{ps}}$ has the Ramsey property. It is also known that $\mathcal{F}_{\text {cen }}$ has the Ramsey property [3, Corollary 2.16]. The celebrated Hindman theorem [25] says that $\mathcal{F}_{\text {ip }}$ has the Ramsey property. This leads to the following questions:

Question 3.11. Is there any family which has the Ramsey property but not the dynamical Ramsey property? 
Question 3.12. Do the families $\mathcal{F}_{\mathrm{ps}}$ and $\mathcal{F}_{\text {ip }}$ have the dynamical Ramsey property?

To end the section we shall discuss 1-independence for various families. Denote by $\mathcal{F}_{\mathrm{rs}}$ the family generated by $\left\{n \mathbb{Z}_{+}: n \in \mathbb{N}\right\}$. The following notion was introduced in 31. Let $(X, T)$ be a t.d.s. We say that $(X, T)$ has dense small periodic sets if for any non-empty open subset $U$ of $X$ there exist a non-empty closed $A \subseteq U$ and $k \in \mathbb{N}$ such that $T^{k} A \subseteq A$. To state our result we need a local version of this notion. That is, for a point $x$ in a t.d.s. $(X, T), x$ is called quasi-regular if for each neighborhood $U$ of $x$, there exist a non-empty closed $A \subseteq U$ and $k \in \mathbb{N}$ such that $T^{k} A \subseteq A$. The closed set of quasi-regular points of $T$ is denoted by $\operatorname{QR}(T)$.

Theorem 3.13. Let $(X, T)$ be a t.d.s. Then

(1) $x \in X$ is $\mathcal{F}_{\text {ip }}$-independent iff $x \in \overline{\operatorname{Rec}(T)}$, where $\operatorname{Rec}(T)$ denotes the set of recurrent points of $T$. Thus, $(X, T)$ is $\mathcal{F}_{\mathrm{ip}}$-independent of order 1 iff $\overline{\operatorname{Rec}(T)}=X$.

(2) $x \in X$ is $\mathcal{F}_{\text {inf }}$-independent iff $x \in \overline{\Lambda(T)}$, where $\Lambda(T)=\bigcup_{x \in X} \omega(x, T)$ and $w(x, T)=\bigcap_{k \geq 0} \overline{\bigcup_{n \geq k}\left\{T^{n} x\right\}}$. Thus, $(X, T)$ is $\mathcal{F}_{\text {inf }}$-independent of order 1 iff $\overline{\Lambda(T)}=X$, iff $\overline{\operatorname{Rec}(T)}=X$.

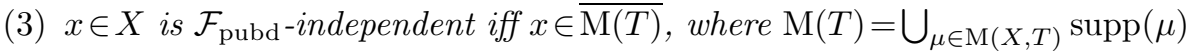
and $\mathrm{M}(X, T)$ denotes the set of all invariant Borel probability measures on

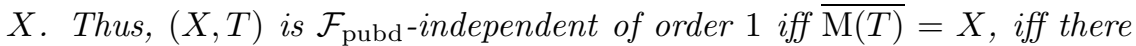
exists a $\mu \in \mathrm{M}(X, T)$ with full support.

(4) $x \in X$ is $\mathcal{F}_{\mathrm{ps}}$-independent if $x \in \overline{\mathrm{AP}(T)}$, where $\mathrm{AP}(T)$ denotes the set of minimal points of $T$. Thus, $(X, T)$ is $\mathcal{F}_{\mathrm{ps}}$-independent of order 1 iff $\overline{\mathrm{AP}(T)}=X$.

(5) $x \in X$ is $\mathcal{F}_{\mathrm{rs}}$-independent iff $x \in \mathrm{QR}(T)$. Thus, $(X, T)$ is $\mathcal{F}_{\mathrm{rs}}$-independent of order 1 iff $\mathrm{QR}(T)=X$.

Proof. (1) Assume that $x \in X$ is $\mathcal{F}_{\text {ip }}$-independent and $U$ is a closed neighborhood of $x$. Then $\operatorname{Ind}(U) \cap \mathcal{F}_{\text {ip }} \neq \emptyset$, and hence there are an IP-set $F$ and $y \in X$ such that $T^{i} y \in U$ for each $i \in F$. By [9, Theorem 5], $U \cap \operatorname{Rec}(T) \neq \emptyset$, i.e. $x \in \overline{\operatorname{Rec}(T)}$.

Conversely, assume that $x \in \overline{\operatorname{Rec}(T)}$ and $U$ is an open neighborhood of $x$. Then there exists a $y \in \operatorname{Rec}(T) \cap U$. By [13, Theorem 2.17], the set $N(y, U)$ contains an IP-set. Thus $\operatorname{Ind}(U) \cap \mathcal{F}_{\text {ip }} \neq \emptyset$.

(2) The first statement follows easily from the definition. The statement that

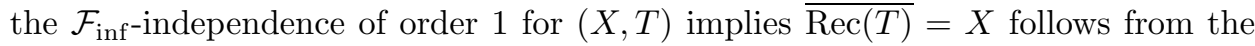
fact that if $(X, T)$ is non-wandering in the sense that $\mathbb{N} \cap N(U, U) \neq \emptyset$ for every non-empty open subset $U$ of $X$, then $\overline{\operatorname{Rec}(T)}=X$ [13, Theorem 1.27].

(3) This was proved in [35, Proposition 3.12].

(4) Assume that $x \in X$ is $\mathcal{F}_{\mathrm{ps}}$-independent and $U$ is a closed neighborhood of $x$. Then $\operatorname{Ind}(U) \cap \mathcal{F}_{\mathrm{ps}} \neq \emptyset$, and hence there are a piecewise syndetic set $F$ and $y \in X$ such that $T^{i} y \in U$ for each $i \in F$. By [9, Theorem 6], $U \cap \operatorname{AP}(T) \neq \emptyset$, i.e. $x \in \overline{\mathrm{AP}(T)}$.

Conversely, assume that $x \in \overline{\mathrm{AP}(T)}$ and $U$ is an open neighborhood of $x$. Then there is $y \in \operatorname{AP}(T) \cap U$. By a well-known result of Gottschalk, $N(y, U)$ contains a syndetic set. Thus $\operatorname{Ind}(U) \cap \mathcal{F}_{\mathrm{ps}} \neq \emptyset$.

(5) It is clear that if $x \in \mathrm{QR}(T)$, then $x$ is an $\mathcal{F}_{\mathrm{rs}}$-independent point. Assume now that $x$ is an $\mathcal{F}_{\text {rs }}$-independent point. Let $U$ be a closed neighborhood of $x$. 
Then there exists a $k \in \mathbb{N}$ such that $k \mathbb{Z}_{+}$is in $\operatorname{Ind}(U)$. Take $z \in \bigcap_{n \in \mathbb{Z}_{+}} T^{-k n} U$. Then $T^{k n} z \in U$ for all $n \in \mathbb{Z}_{+}$. Thus $A:=\overline{\left\{T^{k n} z: n \in \mathbb{Z}_{+}\right\}}$is contained in $U$. It is clear that $T^{k} A \subseteq A$.

Remark 3.14. The family $b \mathcal{F}_{\text {rs }}$ does not have the Ramsey property.

Proof. Let $(X, T)$ be a non-trivial totally minimal t.d.s., i.e., $X$ is minimal under $T^{k}$ for every $k \in \mathbb{N}$. For example, any minimal $(X, T)$ with $X$ a connected topological space is totally minimal [50, $\mathrm{II}(9.6) 8]$. Let $U$ be a non-empty open subset of $X$ with $\bar{U} \neq X$. Then $X=X_{1} \cup X_{2}$ with $X_{1}=\bar{U}$ and $X_{2}=X \backslash U$. Let $y \in X$. We claim that $N\left(y, X_{i}\right) \notin b \mathcal{F}_{\text {rs }}$ for each $i=1,2$. Assume to the contrary that $N\left(y, X_{1}\right) \in b \mathcal{F}_{\mathrm{rs}}$. This means that there are $d \in \mathbb{N}$ and a sequence $\left\{n_{i}\right\}_{i \in \mathbb{N}}$ in $\mathbb{Z}_{+}$ such that for each $i, T^{n_{i}+d j}(y) \in X_{1}$ for each $0 \leq j \leq i$. Replacing $\left\{n_{i}\right\}_{i \in \mathbb{N}}$ by a subsequence if necessary, we may assume that $T^{n_{i}}(y)$ converges to some $z \in X$. Then $z \in X_{1}$ and $T^{d j}(z) \in X_{1}$ for each $j \in \mathbb{N}$, contradicting the assumption that $(X, T)$ is totally minimal. The same argument shows that $N\left(y, X_{2}\right) \notin b \mathcal{F}_{\mathrm{rs}}$. Since $\mathbb{Z}_{+}=N(y, X)=N\left(y, X_{1}\right) \cup N\left(y, X_{2}\right)$, we conclude that $b \mathcal{F}_{\text {rs }}$ does not have the Ramsey property.

\section{Independence: Measurable Case}

In this section, for a given family $\mathcal{F}$, we define $\mathcal{F}$-independence for m.d.s. and discuss 1-independence for various families. First we define independence sets for m.d.s., similar to that for t.d.s. in Definition 3.1

Definition 4.1. Let $(X, \mathcal{B}, \mu, T)$ be an m.d.s. For a tuple $\mathbf{A}=\left(A_{1}, \ldots, A_{k}\right)$ of sets in $\mathcal{B}$, we say that a subset $F \subseteq \mathbb{Z}_{+}$is an independence set for $\mathbf{A}$ if for any non-empty finite subset $J \subseteq F$, we have

$$
\mu\left(\bigcap_{j \in J} T^{-j} A_{s(j)}\right)>0
$$

for any $s \in\{1, \ldots, k\}^{J}$.

We shall still denote the collection of all independence sets for $\mathbf{A}$ by $\operatorname{Ind}\left(A_{1}, \ldots\right.$, $\left.A_{k}\right)$ or IndA. Note that Lemma 3.2 (1)-(3) also holds for m.d.s.

Proposition 4.2. Let $\mathcal{F}$ be a family with the dynamical Ramsey property. For any m.d.s. $(X, \mathcal{B}, \mu, T)$, any $k \in \mathbb{N}$ and $A_{1}, A_{2}, \ldots, A_{k}, A_{1,1}, A_{1,2} \in \mathcal{B}$ with $A_{1}=$ $A_{1,1} \cup A_{1,2}$, if $\operatorname{Ind}\left(A_{1}, A_{2}, \ldots, A_{k}\right) \cap \mathcal{F} \neq \emptyset$, then either $\operatorname{Ind}\left(A_{1,1}, A_{2}, \ldots, A_{k}\right) \cap \mathcal{F} \neq \emptyset$ or $\operatorname{Ind}\left(A_{1,2}, A_{2}, \ldots, A_{k}\right) \cap \mathcal{F} \neq \emptyset$.

Proof. Set $B_{k+1}=X, B_{0}=A_{1,1}, B_{1}=A_{1,2}$, and $B_{i}=A_{i}$ for $2 \leq i \leq k$. Denote by $Y$ the set of elements $s$ in $\Sigma_{k+2}:=\{0,1, \ldots, k+1\}^{\mathbb{Z}_{+}}$satisfying that for any non-empty finite subset $J$ of $\mathbb{Z}_{+}, \mu\left(\bigcap_{j \in J} T^{-j} A_{s(j)}\right)>0$. Then $Y$ is a closed subset of $\Sigma_{k+2}$ and contains the constant function $k+1$. It is also easily checked that $\sigma(Y)=Y$, where $\sigma$ denotes the shift map. Thus $(Y, \sigma)$ is a t.d.s. Note that $\operatorname{Ind}\left(A_{1}, A_{2}, \ldots, A_{k}\right)=\operatorname{Ind}\left([0]_{Y} \cup[1]_{Y},[2]_{Y}, \ldots,[k]_{Y}\right), \operatorname{Ind}\left(A_{1,1}, A_{2}, \ldots, A_{k}\right)=$ $\operatorname{Ind}\left([0]_{Y},[2]_{Y}, \ldots,[k]_{Y}\right)$, and $\operatorname{Ind}\left(A_{1,2}, A_{2}, \ldots, A_{k}\right)=\operatorname{Ind}\left([1]_{Y},[2]_{Y}, \ldots,[k]_{Y}\right)$. Thus $\operatorname{Ind}\left([0]_{Y} \cup[1]_{Y},[2]_{Y}, \ldots,[k]_{Y}\right) \cap \mathcal{F} \neq \emptyset$. Since $\mathcal{F}$ has the dynamical Ramsey property, either $\operatorname{Ind}\left([0]_{Y},[2]_{Y}, \ldots,[k]_{Y}\right) \cap \mathcal{F} \neq \emptyset$ or $\operatorname{Ind}\left([1]_{Y},[2]_{Y}, \ldots,[k]_{Y}\right) \cap \mathcal{F} \neq \emptyset$. That is, either $\operatorname{Ind}\left(A_{1,1}, A_{2}, \ldots, A_{k}\right) \cap \mathcal{F} \neq \emptyset$ or $\operatorname{Ind}\left(A_{1,2}, A_{2}, \ldots, A_{k}\right) \cap \mathcal{F} \neq \emptyset$. 
Next we define $\mathcal{F}$-independence for m.d.s., similar to that for t.d.s. in Definition 3.4 .

Definition 4.3. Let $\mathcal{F}$ be a family and $k \in \mathbb{N}$. We say that an m.d.s. $(X, \mathcal{B}, \mu, T)$ is $\mathcal{F}$-independent of order $k$ if for each tuple $\left(A_{1}, \ldots, A_{k}\right)$ of sets in $\mathcal{B}$ with positive measures, $\operatorname{Ind}\left(A_{1}, \ldots, A_{n}\right) \cap \mathcal{F} \neq \emptyset$. It is said to be $\mathcal{F}$-independent if it is $\mathcal{F}$ independent of order $k$ for each $k \in \mathbb{N}$.

Note that Proposition 3.7(1) holds also for m.d.s.

Remark 4.4. Given a probability space $(X, \mathcal{B}, \mu)$, one may consider the equivalence relation defined on $\mathcal{B}$ by $A \sim B$ exactly when $\mu(A \Delta B)=0$, where $A \Delta B=(A \backslash$ $B) \cup(B \backslash A)$ is the symmetric difference of $A$ and $B$. The set of equivalence classes in $\mathcal{B}$, denoted by $\tilde{\mathcal{B}}$, has the induced operation of taking complement and countable union. Furthermore, $\mu$ descends to a function $\tilde{\mu}$ on $\tilde{\mathcal{B}}$. The pair $(\tilde{B}, \tilde{\mu})$ is called a measure algebra [13, Section 5.1], [14, Section 2.1]. Given a measurable and measure-preserving map $T: X \rightarrow X$, one also gets an induced map $\widetilde{T^{-1}}: \tilde{\mathcal{B}} \rightarrow \tilde{\mathcal{B}}$ preserving $\tilde{\mu}$, complement and countable union. For any family $\mathcal{F}$ and $k \in \mathbb{N}$, it is clear that whether an m.d.s. $(X, \mathcal{B}, \mu, T)$ is $\mathcal{F}$-independent of order $k$ or not depends only on the triple $\left(\tilde{\mathcal{B}}, \tilde{\mu}, \widetilde{T^{-1}}\right)$.

Consider an m.d.s. $(X, \mathcal{B}, \mu, T)$ or a t.d.s. $(X, T)$. Let $\mathbf{A}=\left(A_{1}, \ldots, A_{n}\right)$ be a tuple of subsets of $X$ (in $\mathcal{B}$ for m.d.s.). For each $k \in \mathbb{N}$ set $a_{k}=\max _{F \in \operatorname{Ind} \mathbf{A}} \mid F \cap$ $[0, k-1] \mid$. Then the function $k \mapsto a_{k}$ on $\mathbb{N}$ is subadditive in the sense that $a_{k+j} \leq$

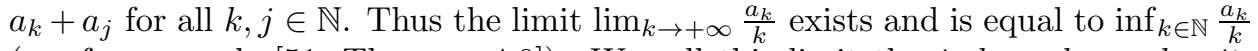
(see for example [51, Theorem 4.9]). We call this limit the independence density of $\mathbf{A}$ and denote it by $I(\mathbf{A})$ (see the discussion before Proposition 3.23 in [35] for the case of actions of discrete amenable groups). The following lemma was proved by Glasner and Weiss in the second paragraph of the proof of Theorem 3.2 in [20, using Birkhoff's ergodic theorem. We give a topological proof here.

Lemma 4.5. There exists $F \in \operatorname{Ind} \mathbf{A}$ with $d(F)=I(\mathbf{A})$.

Proof. For each $k \in \mathbb{N}$ we claim that there exists $F_{k} \in \operatorname{Ind} \mathbf{A}$ such that $F_{k} \subseteq$ $[0, k-1]$ and $\left|F_{k} \cap[0, j-1]\right| \geq j\left(I(\mathbf{A})-\frac{1}{k}\right)$ for all $1 \leq j \leq k$. Suppose that this is not true. Then $I(\mathbf{A})-\frac{1}{k}>0$. Furthermore, for any $F \in \operatorname{Ind} \mathbf{A}$ we can find a strictly increasing sequence $\left\{b_{i}\right\}_{i \in \mathbb{N}}$ in $\mathbb{Z}_{+}$such that $b_{1}=0$, and $b_{i+1}-b_{i} \leq k$ and $\left|F \cap\left[b_{i}, b_{i+1}\right)\right|<\left(b_{i+1}-b_{i}\right)\left(I(\mathbf{A})-\frac{1}{k}\right)$ for all $i \in \mathbb{N}$. Set $m=k^{2}+1$. Take $F \in \operatorname{Ind} \mathbf{A}$ with $|F \cap[0, m-1]|=a_{m}$, and let $\left\{b_{i}\right\}_{i \in \mathbb{N}}$ be as above. Then $b_{s}<m \leq b_{s+1}$ for some $s \in \mathbb{N}$. Thus

$$
\begin{aligned}
m \cdot I(\mathbf{A}) & \leq a_{m}=|F \cap[0, m-1]|=\left|F \cap\left[b_{s}, m-1\right]\right|+\sum_{i=1}^{s-1}\left|F \cap\left[b_{i}, b_{i+1}\right)\right| \\
& \leq k+\sum_{i=1}^{s-1}\left(b_{i+1}-b_{i}\right)\left(I(\mathbf{A})-\frac{1}{k}\right) \leq k+m\left(I(\mathbf{A})-\frac{1}{k}\right),
\end{aligned}
$$

which contradicts $m=k^{2}+1$. This proves our claim.

Now some subsequence of $\left\{1_{F_{k}}\right\}_{k \in \mathbb{N}}$ converges in $\{0,1\}^{\mathbb{Z}_{+}}$to $1_{F}$ for some $F \in \mathbb{Z}_{+}$. Clearly $F \in \operatorname{Ind} \mathbf{A}$ and $|F \cap[0, k-1]| \geq k \cdot I(\mathbf{A})$ for every $k \in \mathbb{N}$. We also have $\lim \sup _{k \rightarrow+\infty} \frac{|F \cap[0, k-1]|}{k} \leq \lim _{k \rightarrow+\infty} \frac{a_{k}}{k}=I(\mathbf{A})$. Therefore $F$ has density $I(\mathbf{A})$. 
We now discuss 1-independence for various families. Using Birkhoff's ergodic theorem, Bergelsen proved part (1) of the following theorem [2, Theorem 1.2]. Here we give a different proof.

Theorem 4.6. Let $(X, \mathcal{B}, \mu, T)$ be an m.d.s. The following hold:

(1) For any $A \in \mathcal{B}$ with $\mu(A)>0$, there exists $F \in \mathcal{F}_{\mathrm{pd}} \cap \operatorname{Ind}(A)$ with density at least $\mu(A)$. In particular, $(X, \mathcal{B}, \mu, T)$ is $\mathcal{F}_{\mathrm{pd}}$-independent of order 1 .

(2) $(X, \mathcal{B}, \mu, T)$ is $\mathcal{F}_{\mathrm{S}}$-independent of order 1 iff $T$ is a.e. periodic, iff $(X, \mathcal{B}, \mu, T)$ is $\mathcal{F}_{\mathrm{rs}}$-independent of order 1 , iff for each $A \in \mathcal{B}$, a.e. every point of $A$ returns to $A$ syndetically, iff for each $A \in \mathcal{B}$, a.e. every point of $A$ returns to $A$ along $n \mathbb{Z}_{+}$for some $n \in \mathbb{N}$.

(3) Let $(X, \mathcal{B}, \mu, T)$ be an m.d.s. If $A$ is in $\mathcal{B}$ with $\mu\left(\bigcup_{i=0}^{n-1} T^{-i} A\right)=1$ for some $n \in \mathbb{N}$, then $\operatorname{Ind}(A) \cap \mathcal{F}_{\mathrm{s}} \neq \emptyset$.

Proof. (1) For each $k \in \mathbb{N}$ let $a_{k}$ be defined as before Lemma4.5 for $\mathbf{A}=(A)$. Then $\sum_{j=0}^{k-1} 1_{T^{-j} A} \leq a_{k}$ a.e. on $X$. Thus $k \mu(A)=\int_{X} \sum_{j=0}^{k-1} 1_{T^{-j} A} d \mu \leq a_{k}$. It follows that $I(\mathbf{A}) \geq \mu(A)$. By Lemma 4.5 we can find $F \in \operatorname{Ind}(A)$ with $d(F)=I(\mathbf{A}) \geq$ $\mu(A)$.

(2) By Theorem 6.8 the first condition implies the second one. Clearly the second condition implies the third one and the fifth one, the third one implies the first one, and the fifth one implies the fourth one. Thus it suffices to show that the fourth condition implies the first one.

Let $A \in \mathcal{B}$ with $\mu(A)>0$ and assume that a.e. every point of $X$ returns to $A$ syndetically. For each $n \in \mathbb{N}$ set $A_{n}=\bigcap_{j \in \mathbb{Z}_{+}} \bigcup_{i=0}^{n-1} T^{-j-i} A$. Then

$$
\mu\left(X \backslash \bigcup_{n \in \mathbb{N}} A_{n}\right)=0,
$$

and thus there exists $n \in \mathbb{N}$ with $\mu\left(A_{n}\right)>0$. Denote by $N$ the union of the measure zero ones among $\bigcap_{j \in J} T^{-j} A$ for $J$ running over non-empty finite subsets of $\mathbb{Z}_{+}$. Then $\mu(N)=0$, and hence $\mu\left(A_{n} \backslash N\right)=\mu\left(A_{n}\right)>0$. Take $x \in A_{n} \backslash N$. Then there exists $F \in \mathcal{F}_{\mathrm{S}}$ such that for each $j \in F, T^{j} x \in A$. For each non-empty finite subset $J$ of $F, x \in\left(\bigcap_{j \in J} T^{-j} A\right) \backslash N$, thus $\mu\left(\bigcap_{j \in J} T^{-j} A\right)>0$. That is, $F \in \operatorname{Ind}(A)$.

(3) The condition implies that $\mu\left(A_{n}\right)=1$. Thus the conclusion follows from the last paragraph.

\section{Classes of topological $\mathcal{F}$-independence}

5.1. General discussion. In this subsection we characterize $\mathcal{F}_{\text {inf }}$ (resp. $\mathcal{F}_{\text {ip }}$ ) independent t.d.s. in Theorem [5.1, construct a non-trivial topological K-system with a unique minimal point in Example 5.7, and discuss $\mathcal{F}_{\mathrm{rs}}$-independence at the end.

A t.d.s. $(X, T)$ is said to be (topologically) transitive if for any non-empty open subsets $U$ and $V$ of $X, N(U, V)$ is non-empty. It is called weakly mixing if ( $X \times$ $X, T \times T)$ is transitive. The equivalence of conditions (1), (2) and (3) in the following theorem was proved in [35, Theorem 8.6]. Here we strengthen it by adding conditions (4) and (5).

Theorem 5.1. For a t.d.s. $(X, T)$ the following are equivalent:

(1) $(X, T)$ is weakly mixing.

(2) $(X, T)$ is $\mathcal{F}_{\text {inf }}$-independent of order 2. 
(3) $(X, T)$ is $\mathcal{F}_{\text {inf }}$-independent.

(4) $(X, T)$ is $\mathcal{F}_{\text {ip }}$-independent of order 2.

(5) $(X, T)$ is $\mathcal{F}_{\mathrm{ip}}$-independent.

Proof. It is clear that $(5) \Rightarrow(4) \Rightarrow(2)$ and $(5) \Rightarrow(3) \Rightarrow(2)$. The implication $(2) \Rightarrow(1)$ follows from the fact that if for any non-empty open subsets $U$ and $V$ of $X$ one has $N(U, U) \cap N(U, V) \neq \emptyset$, then $(X, T)$ is weakly mixing [40, Lemma]. (40, Lemma] was proved only for invertible t.d.s., but it is easy to modify the proof to make it work for any t.d.s.) Thus it suffices to show that $(1) \Rightarrow(5)$.

Now assume that $(X, T)$ is weakly mixing. Then each $(X \times \cdots \times X, T \times \cdots \times T)$ is transitive [12, Proposition II.3]. Thus, for any $n \in \mathbb{N}$, if $U_{1}, \ldots, U_{n}$ and $V_{1}, \ldots, V_{n}$ are non-empty open subsets of $X$, then $\mathbb{N} \cap\left(\bigcap_{i=1}^{n} N\left(U_{i}, V_{i}\right)\right) \neq \emptyset$. For any given non-empty open subsets $U_{1}, \ldots, U_{n}$ of $X$, we are going to find an IP-set $F$ in $\operatorname{Ind}\left(U_{1}, \ldots, U_{n}\right)$.

First there exists a $t_{1} \in \mathbb{N}$ such that $t_{1} \in \bigcap_{\left(i_{1}, i_{2}\right) \in\{1, \ldots, n\}^{2}} N\left(U_{i_{1}}, U_{i_{2}}\right)$. Assume that $t_{1}, \ldots, t_{k}$ in $\mathbb{N}$ are defined such that $\left\{a_{1}, \ldots, a_{j}\right\}:=\{0\} \cup\left\{t_{i_{1}}+\cdots+t_{i_{l}}: 1 \leq\right.$ $\left.i_{1}<\cdots<i_{l} \leq k\right\}$ is in $\operatorname{Ind}\left(U_{1}, \ldots, U_{n}\right)$. Pick $t_{k+1} \in \mathbb{N}$ such that

$$
t_{k+1} \in \bigcap_{1 \leq i_{m} \leq n, 1 \leq m \leq 2 j} N\left(T^{-a_{1}} U_{i_{1}} \cap \cdots \cap T^{-a_{j}} U_{i_{j}}, T^{-a_{1}} U_{i_{j+1}} \cap \cdots \cap T^{-a_{j}} U_{i_{2 j}}\right) .
$$

Then $\{0\} \cup\left\{t_{i_{1}}+\cdots+t_{i_{l}}: 1 \leq i_{1}<\cdots<i_{l} \leq k+1\right\}=\left\{a_{1}, \ldots, a_{j}\right\} \cup\left\{a_{1}+\right.$ $\left.t_{k+1}, \ldots, a_{j}+t_{k+1}\right\}$ is in $\operatorname{Ind}\left(U_{1}, \ldots, U_{n}\right)$. This implies that the IP-set generated by the sequence $\left\{t_{i}\right\}_{i \in \mathbb{N}}$ is in $\operatorname{Ind}\left(U_{1}, \ldots, U_{n}\right)$.

Petersen [41] showed that there exists a t.d.s. which is strictly ergodic, strongly mixing, and has zero topological entropy. Thus in such a system every tuple is $\mathcal{F}_{\text {ip }}$-independent, while no non-diagonal tuple is $\mathcal{F}_{\text {pd }}$-independent.

A t.d.s. is called an E-system if it is transitive and has an invariant Borel probability measure with full support. It is called an $M$-system if it is transitive and the set of minimal points is dense, it is called totally transitive if $\left(X, T^{k}\right)$ is transitive for every $k \in \mathbb{N}$. By Theorems 3.13 and 5.1 we have

Corollary 5.2. Let $(X, T)$ be a t.d.s. The following hold:

(1) If $(X, T)$ is $\mathcal{F}_{\mathrm{pd}}$-independent of order 2 , then it is an E-system.

(2) If $(X, T)$ is $\mathcal{F}_{\mathrm{ps}}$-independent of order 2 , then it is an $M$-system.

(3) If $(X, T)$ is $\mathcal{F}_{\mathrm{rs}}$-independent of order 1 , then it has dense small periodic sets. If it is $\mathcal{F}_{\mathrm{rs}}$-independent of order 2 , then it is totally transitive, and hence is disjoint from all minimal systems by [31, Theorem 3.4].

Definition 5.3. We say that a t.d.s. is topological $K$ if it is $\mathcal{F}_{\text {pubd-independent. }}$

By [32, Theorem 8.3] and [35, Theorem 3.16] a t.d.s. is topological $\mathrm{K}$ if and only if each of its finite covers by non-dense open subsets has positive topological entropy.

Next we show that there is an invertible topological K-system with only one minimal point. Recall that a t.d.s. $(X, T)$ is said to be proximal if the orbit closure of every point in $(X \times X, T \times T)$ has non-empty intersection with the diagonal. 


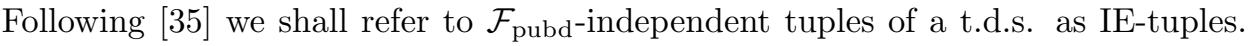
To construct the example we need

Lemma 5.4. Let $(X, T)$ be a t.d.s. We have:

(1) Suppose that $(X, T)$ has a transitive point $x$. Then $T$ is topologically $K$ if and only if for each $j \in \mathbb{N},\left(x, T x, \ldots, T^{j-1} x\right)$ is an IE-tuple.

(2) $(X, T)$ has only one minimal point if and only if $(X, T)$ is proximal.

Proof. (1) This follows from the fact that the set of IE $j$-tuples is closed in $X^{j}$ for each $j \in \mathbb{N}$.

(2) The "only if" part is trivial. Assume that $(X, T)$ is proximal. Take $x \in X$. Say that $(y, y)$ is in the intersection of the diagonal and the orbit closure of $(x, T x)$. Then $T y=y$. Let $z \in X$. Then the orbit closures of $y$ and $z$ have non-empty intersection, which of course has to be $\{y\}$. It follows that if $z$ is minimal, then $z=y$.

For a t.d.s. $(X, T)$, recall its natural extension $(\tilde{X}, \tilde{T})$ defined as follows. $\tilde{X}$ is the closed subspace of $\prod_{n \in \mathbb{N}} X$ consisting of $\left(x_{1}, x_{2}, \ldots\right)$ with $T\left(x_{n+1}\right)=x_{n}$ for all $n \in \mathbb{N}$, and $\tilde{T}$ is defined as $\tilde{T}\left(x_{1}, x_{2}, \ldots\right)=\left(T\left(x_{1}\right), x_{1}, x_{2}, \ldots\right)$. Note that $\tilde{T}$ is a homeomorphism, and the projection $\pi: \tilde{X} \rightarrow X$ sending $\left(x_{1}, x_{2}, \ldots\right)$ to $x_{1}$ is a factor map. It is well known that $(X, T)$ and $(\tilde{X}, \tilde{T})$ share many dynamical properties. Here we need a special case.

Lemma 5.5. Let $(X, T)$ be a t.d.s. The following are true:

(1) Let $\mathcal{F}$ be a family and $k \in \mathbb{N}$. Then $(X, T)$ is $\mathcal{F}$-independent of order $k$ if and only if $(\tilde{X}, \tilde{T})$ is as well.

(2) $(X, T)$ is proximal if and only if $(\tilde{X}, \tilde{T})$ is as well.

Proof. (1) The "if" part follows from the fact that if a t.d.s. is $\mathcal{F}$-independent of order $k$, then so is every factor. Suppose that $(X, T)$ is $\mathcal{F}$-independent of order $k$. Let $U_{1}, \ldots, U_{k}$ be non-empty open subsets of $\tilde{X}$. Then there exist non-empty open subsets $V_{1}, \ldots, V_{k}$ of $X$ and $m \in \mathbb{N}$ such that if $\left(x_{1}, x_{2}, \ldots\right)$ is in $\tilde{X}$ and $x_{m} \in V_{j}$ for some $1 \leq j \leq k$, then $\left(x_{1}, x_{2}, \ldots\right)$ is in $U_{j}$.

We claim that $\operatorname{Ind}\left(V_{1}, \ldots, V_{k}\right) \subseteq \operatorname{Ind}\left(U_{1}, \ldots, U_{k}\right)$. Let $F \in \operatorname{Ind}\left(V_{1}, \ldots, V_{k}\right), J$ be a non-empty finite subset of $F$, and $s \in\{1, \ldots, k\}^{J}$. Then $\bigcap_{j \in J} T^{-j} V_{s(j)} \neq \emptyset$. Take $y \in \bigcap_{j \in J} T^{-j} V_{s(j)}$. We can find $\tilde{x}=\left(x_{1}, x_{2}, \ldots\right) \in \tilde{X}$ such that $x_{m}=y$. Then $\tilde{x} \in \bigcap_{j \in J} \tilde{T}^{-j} U_{s(j)}$. Thus $F \in \operatorname{Ind}\left(U_{1}, \ldots, U_{k}\right)$. This proves our claim.

Since $\mathcal{F} \cap \operatorname{Ind}\left(V_{1}, \ldots, V_{k}\right) \neq \emptyset$, we get $\mathcal{F} \cap \operatorname{Ind}\left(U_{1}, \ldots, U_{k}\right) \neq \emptyset$. Therefore $(\tilde{X}, \tilde{T})$ is also $\mathcal{F}$-independent of order $k$. This proves the "only if" part.

(2) This is trivial.

For $p \geq 2$ let $\Lambda_{p}=\{0,1, \ldots, p-1\}$ with the discrete topology, $\Sigma_{p}=\Lambda_{p}^{\mathbb{Z}_{+}}$ with the product topology and $\sigma: \Sigma_{p} \rightarrow \Sigma_{p}$ be the shift. For $n \in \mathbb{N}$ and $a=$ $(a(1), a(2), \ldots, a(n)) \in \Lambda_{p}^{n}$ (a block of length $\left.n\right)$, let $|a|=n, \sigma(a)=(a(2), \ldots, a(n))$. We say that $a$ appears in $x=(x(1), x(2), \ldots) \in \Sigma_{p}$ or $x \in \Lambda_{p}^{m}$ with $m \geq n$ if there is $j \in \mathbb{N}$ with $a=(x(j), x(j+1), \ldots, x(j+n-1))$ (write $a<x$ for short), and we use $t^{i}$ to denote $t \ldots t$ ( $i$ times). For $b=(b(1), \ldots, b(m)) \in \Lambda_{p}^{m}$, denote $(a(1), \ldots, a(n), b(1), \ldots, b(m)) \in \Lambda_{p}^{n+m}$ by $a b$. Denote $(i i \ldots)$ by $\mathbf{i}, 0 \leq i \leq p-1$. 
We also need the following lemma. In view of [5, Proposition 2] and 32, Theorem 7.3] or [35, Theorem 3.16], it is equivalent to [28, Lemma 4.1]. One can also prove it directly using IE-pairs instead of entropy pairs in the proof of [28, Lemma 4.1].

Lemma 5.6. There is an E-system $(Y, \sigma)$ contained in $\left(\Sigma_{3}, \sigma\right)$ with a unique minimal point $\mathbf{0}$ such that $Y$ has an IE-pair $\left(x_{1}, x_{2}\right)$ in $[1]_{Y} \times[2]_{Y}$.

Example 5.7. There exists a non-trivial invertible t.d.s. which is topological $\mathrm{K}$ and has a unique minimal point.

Proof. By Lemmas 5.4 and 5.5 it suffices to show that there exists a non-trivial t.d.s. which is topological $\mathrm{K}$ and proximal. We use the idea in the proof of Theorem 4.2 in 28. The main idea is to construct a recurrent point $x \in \Sigma_{2}$ with the following two properties:

(I) for any $j \in \mathbb{N},\left(x, \sigma(x), \ldots, \sigma^{j-1}(x)\right)$ is an IE-tuple of $(X, \sigma)$, where $X$ is the orbit closure of $x$, and

(II) for each $n \in \mathbb{N}, 0^{n}$ appears in $x$ syndetically.

By Lemma 5.4 it is clear that $(X, \sigma)$ is topological $\mathrm{K}$ with a unique minimal point $\mathbf{0}$. First we give the detailed construction of the recurrent point $x$.

Let $(Y, \sigma)$ be the system constructed in Lemma 5.6 and let $y$ be a transitive point of $Y$. By [35, Theorem 3.18] for each $m \in \mathbb{N}$ we can find an IE-tuple $\left(z_{m, 1}, \ldots, z_{m, m}\right)$ of $Y$ with $z_{m, 1}, \ldots, z_{m, m}$ pairwise distinct and all in $[1]_{Y} \cup[2]_{Y}$. Then we can find $t_{m} \in \mathbb{N}$ such that $z_{m, 1}\left[0, t_{m}\right], \ldots, z_{m, m}\left[0, t_{m}\right]$ are pairwise distinct, where $z[0, t]$ denotes $(z(0), \ldots, z(t))$. Define a map $f_{m}: \Lambda_{3}^{t_{m}+1} \rightarrow \Lambda_{m+1}$ by $f_{m}(a)=j$ if $a=z_{m, j}\left[0, t_{m}\right]$ for some $1 \leq j \leq m$ and $f_{m}(a)=0$ otherwise.

Take $\phi: \mathbb{N} \rightarrow \mathbb{N}$ such that $\phi(k) \leq k$ for each $k \in \mathbb{N}$ and for each $m \in \mathbb{N}, \phi^{-1}(m)$ is infinite.

Set $A_{1}=(10), n_{1}=\left|A_{1}\right|=2$ and set

$$
C_{1,0}=0^{2 n_{1}}, C_{1,1}=A_{1} 0^{n_{1}} .
$$

Suppose that $A_{1}, \ldots, A_{k}, C_{m, i}$ for $0<m \leq k$ and $0 \leq i \leq m$, and $n_{1}, \ldots, n_{k}$ are defined. We define inductively $A_{k+1}, C_{k+1, i}$ for $i=0,1, \ldots, k+1$, and $n_{k+1}$.

Say that $m=\phi(k)$. Since $(Y, \sigma)$ has a unique minimal point $\mathbf{0}$, there exists $\ell_{k} \in \mathbb{N}$ with $\ell_{k} \geq t_{m}$ such that $0^{n_{k}}$ appears in $y$ with gaps bounded above by $\ell_{k}$. Set $b_{k}=2 \ell_{k} n_{k}$, and set

$$
\begin{aligned}
& \left.A_{k+1}=A_{k} 0^{n_{k}} C_{m, f_{m}\left(y\left[0, t_{m}\right]\right)} C_{m, f_{m}\left(y\left[1, t_{m}+1\right]\right)} \cdots C_{m, f_{m}\left(y\left[b_{k}-t_{m}, b_{k}\right]\right.}\right)^{2 n_{k}}, \\
& n_{k+1}=\left|A_{k+1}\right|, \text { and } \\
& C_{k+1,0}=0^{2 n_{k+1}}, C_{k+1, i}=\sigma^{i-1}\left(A_{k+1}\right) 0^{i-1} 0^{n_{k+1}} \text { for } i=1,2, \ldots, k+1 .
\end{aligned}
$$

It is clear that $x:=\lim _{k \rightarrow+\infty} A_{k}$ is a recurrent point of $\sigma$ in $\Sigma_{2}$. Denote by $X$ the orbit closure of $x$ in $\Sigma_{2}$. We claim that $x$ satisfies (I) and (II).

(I) Given $j \in \mathbb{N}$, we show that $\left(x, \sigma(x), \ldots, \sigma^{j-1}(x)\right)$ is an IE-tuple of $(X, \sigma)$. Suppose that $V_{0}^{\prime}, V_{1}^{\prime}, \ldots, V_{j-1}^{\prime}$ are neighborhoods of $x, \sigma(x), \ldots, \sigma^{j-1}(x)$, respectively. Then there is some $m \in \mathbb{N}$ with $m>j$ such that $V_{i} \subseteq V_{i}^{\prime}$ for all $0 \leq i \leq j-1$, where $V_{i}:=\left[\sigma^{i}\left(A_{m}\right) 0^{i}\right]_{X}$ for all $0 \leq i \leq m-1$.

Since $\left(z_{m, 1}, \ldots, z_{m, m}\right)$ is an IE-tuple of $Y$, there exists some $d>0$ such that for any $n \in \mathbb{N}$ we can find a finite subset $J \subseteq \mathbb{Z}_{+}$with $|J| \geq n$ contained in an interval with length at most $d|J|$ such that for any $s \in\{1,2, \ldots, m\}^{J}$ one has $\bigcap_{i \in J} \sigma^{-i} U_{s(i)} \neq \emptyset$, where $U_{j}=\left(z_{m, j}\left[0, t_{m}\right]\right)_{Y}$ for $1 \leq j \leq m$. Since $y$ is 
a transitive point of $Y$, we have $\sigma^{N}(y) \in \bigcap_{i \in J} \sigma^{-i} U_{s(i)}$ for some $N \in \mathbb{Z}_{+}$. Then $y_{\left[N+i, N+i+t_{m}\right]}=z_{m, s(i)}\left[0, t_{m}\right]$ for all $i \in J$. Take $k \geq N+\max J+t_{m}$ with $\phi(k)=m$. Then $b_{k} \geq k \geq N+i+t_{m}$ for all $i \in J$, and

$$
A_{k+1}=A_{k} 0^{n_{k}} C_{m, f_{m}\left(y\left[0, t_{m}\right]\right)} C_{m, f_{m}\left(y\left[1, t_{m}+1\right]\right)} \cdots C_{m, f_{m}\left(y\left[b_{k}-t_{m}, b_{k}\right]\right)} 0^{2 n_{k}} .
$$

Note that $f_{m}\left(y\left[N+i, N+i+t_{m}\right]\right)=s(i)$ for all $i \in J$. Thus $\sigma^{2 n_{k}+2(N+i) n_{m}}(x) \in$ $\left[C_{m, s(i)}\right]_{X} \subseteq V_{s(i)-1}$ for all $i \in J$. It follows that $\sigma^{2 n_{k}+2 N n_{m}}(x) \in \bigcap_{i \in 2 n_{m} J} \sigma^{-i} V_{\psi(i)}$ for the map $\psi \in\{0,1, \ldots, m-1\}^{2 n_{m} J}$ defined by $\psi\left(2 n_{m} i\right)=s(i)-1$ for all $i \in J$. Therefore $2 n_{m} J$ is an independence set for $\left(V_{0}, \ldots, V_{m-1}\right)$. Clearly $2 n_{m} J$ is contained in an interval with length at most $2 n_{m} d|J|=2 n_{m} d\left|2 n_{m} J\right|$. Thus by Lemma $4.5\left(x, \sigma(x), \ldots, \sigma^{j-1}(x)\right)$ is an IE-tuple of $(X, \sigma)$.

(II) We now show that for each $n \in \mathbb{N}, 0^{n}$ appears in $x$ syndetically. It suffices to prove that for each $k \in \mathbb{N}, 0^{n_{k}}$ appears in $x$ syndetically with gaps bounded above by $2 b_{k}$.

Fix $k \in \mathbb{N}$. Say that $\phi(k)=m$. By the construction,

$$
A_{k+1}=A_{k} 0^{n_{k}} C_{m, f_{m}\left(y\left[0, t_{m}\right]\right)} C_{m, f_{m}\left(y\left[1, t_{m}+1\right]\right)} \cdots C_{m, f_{m}\left(y\left[b_{k}-t_{m}, b_{k}\right]\right)} 0^{2 n_{k}} .
$$

Note that $f_{m}(a)=0$ for every $a \in \Lambda_{3}^{t_{m}+1}$ with $a(0)=0$. As $0^{n_{k}}$ appears in $y$ with gaps bounded above by $\ell_{k}, 0^{n_{k}}$ appears in $C_{m, f_{m}\left(y\left[0, t_{m}\right]\right)} C_{m, f_{m}\left(y\left[1, t_{m}+1\right]\right)} \cdots$ $C_{m, f_{m}\left(y\left[b_{k}-t_{m}, b_{k}\right]\right)}$ with gaps bounded above by $2 n_{m} \ell_{k} \leq 2 n_{k} \ell_{k}=b_{k}$. Thus $0^{n_{k}}$ appears in $A_{k+1}$ with gaps bounded above by $b_{k}+n_{k} \leq 2 b_{k}$.

Assume that $0^{n_{k}}$ appears in $A_{\ell}$ with gaps bounded above by $2 b_{k}$, where $\ell \geq k+1$. Now we are going to prove that this is also true for $\ell+1$. Set $m^{\prime}=\phi(\ell)$. First note that

$$
A_{\ell+1}=A_{\ell} 0^{n_{\ell}} C_{m^{\prime}, f_{m^{\prime}}\left(y\left[0, t_{m^{\prime}}\right]\right)} C_{m^{\prime}, f_{m^{\prime}}\left(y\left[1, t_{m^{\prime}}+1\right]\right)} \cdots C_{m^{\prime}, f_{m^{\prime}}\left(y\left[b_{\ell}-t_{m^{\prime}}, b_{\ell}\right]\right)} 0^{2 n_{\ell}} .
$$

If $m^{\prime} \geq k+1$, then by the induction assumption and the construction of $C_{m^{\prime}, i}$, we know that $0^{n_{k}}$ appears in $A_{\ell+1}$ with gaps bounded above by $2 b_{k}$. If $m^{\prime} \leq k$, then by the induction assumption and the discussion similar to the case of $A_{k+1}$, we know that $0^{n_{k}}$ appears in $A_{\ell+1}$ with gaps bounded above by $2 b_{k}$. Hence $0^{n_{k}}$ appears in $x$ syndetically with gaps bounded above by $2 b_{k}$, as $x=\lim _{\ell \rightarrow+\infty} A_{\ell}$.

Definition 5.8. We say that a t.d.s $(X, T)$ is Bernoulli if it is conjugate to $\left(A^{\mathbb{Z}_{+}}, \sigma\right)$, where $A$ is a compact metrizable space with $|A| \geq 2$ and $\sigma$ is the shift.

Theorem 5.9. A Bernoulli system is $\mathcal{F}_{\mathrm{rs}}$-independent.

Proof. Let $(X, T)$ be a Bernoulli system. Without loss of generality we may assume that $(X, T)=\left(A^{\mathbb{Z}_{+}}, \sigma\right)$ as above. Let $U_{1}, \ldots, U_{n}$ be non-empty open subsets of $X$ for some $n \in \mathbb{N}$. Then there exist some $k \in \mathbb{N}$ and non-empty subsets $A_{i, j} \subseteq A$ for $1 \leq i \leq n$ and $0 \leq j<k$ such that $U_{i} \supseteq A_{i, 0} \times \cdots \times A_{i, k-1} \times \prod_{\ell \geq k} A$ for all $1 \leq i \leq n$. It follows that $k \mathbb{N} \subseteq \operatorname{Ind}\left(U_{1}, \ldots, U_{n}\right)$. Thus $(X, T)$ is $\mathcal{F}_{\mathrm{rs}}$-independent.

Recall that a t.d.s. $(X, T)$ is called strongly mixing if for any non-empty open subsets $U$ and $V$ of $X, N(U, V)$ is a cofinite subset of $\mathbb{Z}_{+}$. In [4, Example 5] Blanchard constructed examples of invertible t.d.s. which are $\mathcal{F}_{\text {rs }}$-independent of order 2 and are not strongly mixing. In fact, the Property $\mathrm{P}$ defined in [4] is exactly the same as $\mathcal{F}_{\mathrm{rs}}$-independence of order 2 . It is easily checked that the condition in [4. Proposition 4] actually implies $\mathcal{F}_{\mathrm{rs}}$-independence. Thus Blanchard's examples are actually $\mathcal{F}_{\mathrm{rs}}$-independent. Thus $\mathcal{F}_{\mathrm{rs}}$-independence does not imply strong mixing and hence does not imply Bernoulli. 
A factor map $\pi:(X, T) \rightarrow(Y, S)$ between t.d.s. is said to be an almost one-toone extension if the set $\left\{x \in X: \pi^{-1}(\pi(x))=\{x\}\right\}$ is dense in $X$.

For a sequence $K=\left\{k_{n}\right\}_{n \in \mathbb{N}}$ in $\mathbb{N}$ with $k_{n+1}$ being divisible by $k_{n}$ for each $n \in \mathbb{N}$, the adding machine $\left(X_{K}, T_{K}\right)$ associated to $K$ is defined as follows. $X_{K}$ is the projective limit of $\lim _{n \rightarrow+\infty} \mathbb{Z} / k_{n} \mathbb{Z}$, as a metrizable compact abelian group, and $T_{K}$ is the addition by 1 .

For a t.d.s. $(X, T)$, recall that $x \in X$ is called a regular minimal point 23 , Definition 3.38] if for each neighborhood $U$ of $x$, there exists $k \in \mathbb{N}$ such that $N(x, U) \supseteq k \mathbb{Z}_{+}$. It is known that if $x$ is a regular minimal point, then its orbit closure is an almost one-to-one extension of some adding machine; see for instance [31, Proposition 3.5]. Now we show

Proposition 5.10. Let $(X, T)$ be a minimal t.d.s. The following are equivalent:

(1) $(X, T)$ has dense small periodic sets.

(2) $(X, T)$ is an almost one-to-one extension of some adding machine.

(3) $X$ has a regular minimal point.

Proof. By [31, Proposition 3.5] (2) and (3) are equivalent. $(3) \Rightarrow(1)$ is trivial.

$(1) \Rightarrow(2)$. For any non-empty open subset $U$ of $X$, let $B$ be a non-empty closed subset of $U$ with $T^{k} B \subseteq B$ for some $k \in \mathbb{N}$. Take $x \in B$. Then the argument in the proof of [31, Proposition 3.5] shows that the orbit closure $A$ of $x$ under $T^{k}$ is a nonempty clopen subset of $U$ and there exists some $\ell \in \mathbb{N}$ such that $\left\{A, T A, \ldots, T^{\ell-1} A\right\}$ is a clopen partition of $X$ and $T^{\ell} A=A$.

Fix a compatible metric on $X$. Starting with some non-empty open subset $U$ of $X$ with $\operatorname{diam}(U)<1$, we obtain $A$ and $\ell$ as above, and set $A_{1}=A$ and $\ell_{1}=\ell$. Inductively, assuming that we have found subsets $A_{1} \supseteq A_{2} \supseteq \cdots \supseteq A_{k}$ and positive integers $\ell_{1}, \ell_{2}, \ldots, \ell_{k}$ such that $\operatorname{diam}\left(A_{j}\right)<1 / j,\left\{A_{j}, T A_{j}, \ldots, T^{\ell_{j}-1} A_{j}\right\}$ is a clopen partition of $X$ and $T^{\ell_{j}} A_{j}=A_{j}$ for all $1 \leq j \leq k$. We shall find $A_{k+1}$ and $\ell_{k+1}$ with the same property. Let $U$ be a non-empty open subset of $A_{k}$ with $\operatorname{diam}(U)<1 /(k+1)$. We obtain $A$ and $\ell$ as above and set $A_{k+1}=A$ and $\ell_{k+1}=\ell$.

Now the argument in the proof of [31, Proposition 3.5] shows that $(X, T)$ is an almost one-to-one extension of some adding machine.

5.2. Non-existence of non-trivial minimal $\mathcal{F}_{\mathrm{s}}$-independent t.d.s. It was shown in [32, Theorem 3.4] that there exist non-trivial minimal topological Ksystems (the existence of non-trivial minimal u.p.e. systems was proved earlier by Glasner and Weiss [18], answering a question of Blanchard [5]). As a contrast, we have

Theorem 5.11. There is no non-trivial minimal t.d.s. which is $\mathcal{F}_{\mathrm{s}}$-independent of order 2 .

To prove Theorem [5.11, we need some preparation. Crucial to the proof of Theorem 5.11 is the following combinatorial result, which is also of independent interest. We postpone its proof to the appendix. Recall the notion introduced before Lemma 5.6

Theorem 5.12. Let $p, \ell \in \mathbb{N}$ with $p \geq 2$. For any integer $m \geq 4 \ell+2$, given any sequence $\left\{A_{n}\right\}_{n \in \mathbb{Z}_{+}}$of subsets of $\Lambda_{p}^{m}$ with $\left|A_{n}\right| \leq \ell$ for each $n \in \mathbb{Z}_{+}$, there exists $x \in \Sigma_{p}$ such that $x[n, n+m-1] \notin A_{n}$ for every $n \in \mathbb{Z}_{+}$. 
We remark that under the conditions of Theorem 5.12 , the set $\left\{x \in \Sigma_{p}: x[n, n+\right.$ $m-1] \notin A_{n}$ for all $\left.n \in \mathbb{Z}_{+}\right\}$is small in both the topological and measure-theoretical senses: it is a closed subset of $\Sigma_{p}$ with empty interior and has measure 0 for the product measure on $\Sigma_{p}$ associated to any probability vector $\left(t_{0}, \ldots, t_{p-1}\right)$ with $\sum_{j=0}^{p-1} t_{j}=1$ and $t_{j}>0$ for all $0 \leq j \leq k-1$. The following lemma is important for the proof of Theorem 5.11 and can also be applied to show that an $\mathcal{F}_{\mathrm{s}}$-independent t.d.s. is disjoint from all minimal t.d.s. [11].

Lemma 5.13. For every minimal subshift $X \subseteq \Sigma_{2}$, Ind $\left([0]_{X},[1]_{X}\right)$ does not contain any syndetic set.

Proof. We argue by contradiction. Assume that $X \subseteq \Sigma_{2}$ is a minimal subshift and $\operatorname{Ind}\left([0]_{X},[1]_{X}\right)$ contains a syndetic set $F$. Say $F=\left\{n_{0}<n_{1}<\cdots\right\}$ with $\ell=\max _{j \in \mathbb{Z}_{+}}\left(n_{j+1}-n_{j}\right)$. Let $m$ be as in Theorem 5.12 for $p=2$ and $\ell$. Take $a \in \Lambda_{2}^{m \ell}$ such that $a$ appears in some element of $X$. For each $j \in \mathbb{Z}_{+}$, set $A_{j}$ to be the subset of $\Lambda_{2}^{m}$ consisting of elements of the form $\left(a(k), a\left(k+n_{j+1}-n_{j}\right), a(k+\right.$ $\left.\left.n_{j+2}-n_{j}\right), \ldots, a\left(k+n_{j+m-1}-n_{j}\right)\right)$ for $1 \leq k \leq \ell$. Then $\left|A_{j}\right| \leq \ell$ for all $j \in \mathbb{Z}_{+}$. By Theorem 5.12 we can find $x \in \Sigma_{2}$ such that $x[j, j+m-1] \notin A_{j}$ for every $j \in \mathbb{Z}_{+}$. Since $F \in \operatorname{Ind}\left([0]_{X},[1]_{X}\right)$, we can find $y \in X$ with $y\left(n_{j}\right)=x(j)$ for all $j \in \mathbb{Z}_{+}$. As $X$ is minimal, there exists some $i \geq n_{1}$ such that $y[i, i+m \ell-1]=a$. Say that $n_{j-1}<i \leq n_{j}$. Set $k=n_{j}-i+1$. Then $x(s)=y\left(n_{s}\right)=a\left(k+n_{s}-n_{j}\right)$ for all $j \leq s \leq j+m-1$, which contradicts the fact that $x[j, j+m-1] \notin A_{j}$.

We are ready to prove Theorem 5.11

Proof of Theorem 5.11. We shall show that if $(Y, S)$ is a minimal t.d.s. and $V_{0}, V_{1}$ are disjoint closed subsets of $X$ with non-empty interior, then $\operatorname{Ind}\left(V_{0}, V_{1}\right)$ does not contain any syndetic set.

It is well known that we can find a minimal t.d.s. $\left(X_{1}, T_{1}\right)$ and a factor map $\pi:\left(X_{1}, T_{1}\right) \rightarrow(Y, S)$ such that $X_{1}$ is a closed subset of a Cantor set (see for example [6, page 34]). It is easy to see that $\operatorname{Ind}\left(V_{0}, V_{1}\right)=\operatorname{Ind}\left(\pi^{-1}\left(V_{0}\right), \pi^{-1}\left(V_{1}\right)\right)$. Write $X$ as the disjoint union of clopen subsets $U_{0}$ and $U_{1}$ such that $U_{j} \supseteq \pi^{-1}\left(V_{j}\right)$ for $j=0,1$. Then $\operatorname{Ind}\left(V_{0}, V_{1}\right) \subseteq \operatorname{Ind}\left(U_{0}, U_{1}\right)$.

Define a coding $\phi: X_{1} \rightarrow \Sigma_{2}$ such that for each $x \in X_{1}, \phi(x)=\left(x_{0}, x_{1}, \ldots\right)$, where $x_{i}=j$ if $T_{1}^{i}(x) \in U_{j}$ for all $i \in \mathbb{Z}_{+}$. Then $X=\phi\left(X_{1}\right)$ is a minimal subshift contained in $\Sigma_{2}$ and $\phi: X_{1} \rightarrow X$ is a factor map. It is easy to verify that $\operatorname{Ind}\left(U_{0}, U_{1}\right) \subseteq \operatorname{Ind}\left([0]_{X},[1]_{X}\right)$.

By Lemma 5.13 we know that $\operatorname{Ind}\left([0]_{X},[1]_{X}\right)$ does not contain any syndetic set. Then $\operatorname{Ind}\left(V_{0}, V_{1}\right)$ does not contain any syndetic set either.

5.3. Finite product. In this subsection we investigate the question as to which families $\mathcal{F}$ the product of finitely many $\mathcal{F}$-independent t.d.s. remains $\mathcal{F}$-independent.

It is known that if $\mathcal{F}=\mathcal{F}_{\mathrm{pd}}$ the question has a positive answer 32, Theorem 8.1], [35, Theorem 3.15]. We now show that the question has a positive answer for $\mathcal{F}=\mathcal{F}_{\text {rs }}, \mathcal{F}_{\text {ps }}$. It is clear that

$$
\mathcal{F}_{\text {rs }} \subseteq \mathcal{F}_{\text {cen }} \subseteq \mathcal{F}_{\text {ps }} \subseteq \mathcal{F}_{\text {pubd }}
$$

We need the following lemma. It is also needed for the proof of Theorem 7.1 later. 
Lemma 5.14. For any $d>0, k \in \mathbb{N}$, and finite subset $F \subseteq \mathbb{Z}_{+}$with $d|F|>k$, there exists $N=N(d, k, F) \in \mathbb{N}$ such that for any non-empty finite interval $I \subseteq \mathbb{Z}_{+}$ and $S \subseteq I$ with $\frac{|S|}{|I|} \geq d$ and $|I| \geq N$, one has $|S \cap(F+p)| \geq k$ for some $p \in \mathbb{Z}$.

Proof. Take $N \in \mathbb{N}$ such that $\frac{d|F|}{1+(\max F) / N} \geq k$. For each $j \in F$ the set $S-j$ is contained in $[\min I-\max F, \max I] \subseteq \mathbb{Z}$. Then we can find some $p \in[\min I-$ $\max F, \max I]$ such that $p$ is contained in $S-j$ for at least $\frac{|S| \cdot|F|}{|[\min I-\max F, \max I]|} j$ 's in $F$. Set $W=\{j \in F: p \in S-j\}$. Then $(W+p) \subseteq S \cap(F+p)$ and

$$
|W| \geq \frac{|S| \cdot|F|}{|[\min I-\max F, \max I]|}=\frac{|S| \cdot|F|}{|I|+\max F} \geq \frac{d|F|}{1+(\max F) /|I|} \geq k .
$$

We need the following simple lemma. For a subset $K$ of $\mathbb{Z}_{+}$, denote by $X_{K}$ the set of limit points of the sequence $\left\{\sigma^{n} 1_{K}\right\}_{n \in \mathbb{Z}_{+}}$in $\{0,1\}^{\mathbb{Z}_{+}}$, where $\sigma$ denotes the shift map on $\{0,1\}^{\mathbb{Z}_{+}}$. Note that $\left(X_{K}, \sigma\right)$ is a t.d.s.

Lemma 5.15. The following statements hold:

(1) Let $S_{1}, S_{2} \in \mathcal{F}_{\text {pubd }}$. Then there are two subsets $K_{1}, K_{2}$ of $\mathbb{Z}_{+}$such that $1_{K_{i}} \in X_{S_{i}}, i=1,2$, and $K_{1} \cap K_{2} \in \mathcal{F}_{\text {pud }}$.

(2) Let $S_{1}, S_{2} \in \mathcal{F}_{\mathrm{ps}}$. Then there are two subsets $K_{1}, K_{2}$ of $\mathbb{Z}_{+}$such that $1_{K_{i}} \in X_{S_{i}}, i=1,2$, and $K_{1} \cap K_{2} \in \mathcal{F}_{\mathrm{s}} \cap \mathcal{F}_{\text {cen }}$.

(3) Let $S_{1}, S_{2} \in \mathcal{F}_{\mathrm{rs}}$. Then there are two subsets $K_{1}, K_{2}$ of $\mathbb{Z}_{+}$such that $1_{K_{i}} \in$ $X_{S_{i}}, i=1,2$, and $K_{1} \cap K_{2} \in \mathcal{F}_{\text {rs }}$.

Proof. (1) Set $X_{i}=X_{S_{i}}$. Recall the independence density defined before Lemma 4.5 . We have $I\left([1]_{X_{i}}\right)=B D^{*}\left(S_{i}\right)>0$ for $i=1,2$. For each $k \in \mathbb{N}$, take a finite interval $J_{1}$ in $\mathbb{Z}_{+}$with $\left|J_{1}\right|=k$ and a set $F_{1} \in \operatorname{Ind}\left([1]_{X_{1}}\right)$ with $F_{1} \subseteq J_{1}$ and $\left|F_{1}\right| \geq$ $\left|J_{1}\right| I\left([1]_{X_{1}}\right)$. Note that we can find an arbitrarily long finite interval $J_{2}$ in $\mathbb{Z}_{+}$and a set $F_{2} \in \operatorname{Ind}\left([1]_{X_{1}}\right)$ with $F_{2} \subseteq J_{2}$ and $\left|F_{2}\right| \geq\left|J_{2}\right| I\left([1]_{X_{2}}\right)$. By Lemma 5.14, when $\left|I_{2}\right|$ is large enough, we have $\left|F_{1} \cap\left(F_{2}+p\right)\right| \geq I\left([1]_{X_{2}}\right)\left|F_{1}\right|-1 \geq\left|J_{1}\right| I\left([1]_{X_{1}}\right) I\left([1]_{X_{2}}\right)-1$ for some $p \in \mathbb{Z}$. Consider the t.d.s. $\left(X_{1} \times X_{2}, \sigma \times \sigma\right)$. Note that $F_{1} \cap\left(F_{2}+p\right) \in$ $\operatorname{Ind}\left([1]_{X_{1}} \times[1]_{X_{2}}\right)$. It follows that $I\left([1]_{X_{1}} \times[1]_{X_{2}}\right) \geq I\left([1]_{X_{1}}\right) I\left([1]_{X_{2}}\right)>0$. By Lemma 4.5 we can find $F \in \operatorname{Ind}\left([1]_{X_{1}} \times[1]_{X_{2}}\right)$ with density $I\left([1]_{X_{1}} \times[1]_{X_{2}}\right)$. Take $x \in \bigcap_{n \in F}(\sigma \times \sigma)^{-n}\left([1]_{X_{1}} \times[1]_{X_{2}}\right)$. Say that $x=\left(1_{K_{1}}, 1_{K_{2}}\right)$ for some $K_{1}, K_{2} \in \mathbb{Z}_{+}$. Then $1_{K_{i}} \in X_{i}, i=1,2$, and $K_{1} \cap K_{2} \supseteq F$. It follows that $K_{1} \cap K_{2} \in \mathcal{F}_{\text {pud }}$.

(2) Set $X_{i}=X_{S_{i}}$. Since $S_{i}$ is in $\mathcal{F}_{\mathrm{ps}}$, there exists $1_{F_{i}} \in X_{i}$ with $F_{i} \in \mathcal{F}_{\mathrm{s}}$. By Lemma $5.4(2)$, for each $i=1,2$, there is a minimal set $M_{i} \neq\{(0,0, \ldots)\}$ contained in $X_{i}$. Consider the t.d.s. $\left(M_{1} \times M_{2}, \sigma \times \sigma\right)$. Let $M$ be a minimal set contained in $M_{1} \times M_{2}$ and take $x \in M$. Say that $x=\left(1_{K_{1}}, 1_{K_{2}}\right)$ for some $K_{1}, K_{2} \subseteq \mathbb{Z}_{+}$. Then $K_{1}$ and $K_{2}$ are non-empty. For any $j, k \in \mathbb{Z}_{+}$, since $\sigma^{j} \times \sigma^{k}$ is a factor map from $M$ to a minimal set in $M_{1} \times M_{2}, \sigma^{j} \times \sigma^{k}(x)=\left(\sigma^{j} 1_{K_{1}}, \sigma^{k} 1_{K_{2}}\right)$ is also a minimal point. Replacing $x$ by $\sigma^{\min K_{1}} \times \sigma^{\min K_{2}}(x)$ if necessary, we may assume that $\min K_{1}=\min K_{2}=0$. Then $K_{1} \cap K_{2}=N\left(x,[1]_{X_{1}} \times[1]_{X_{2}}\right)$ is syndetic and central.

(3) This is trivial.

Theorem 5.16. The product of finitely many $\mathcal{F}_{\mathrm{s}}-\left(\right.$ resp. $\left.\mathcal{F}_{\mathrm{rs}^{-}}, \mathcal{F}_{\mathrm{pd}^{-}}{ }^{-}\right)$independent t.d.s. is $\mathcal{F}_{\mathrm{s}^{-}}\left(\right.$resp. $\left.\mathcal{F}_{\mathrm{rs}^{-}}, \mathcal{F}_{\mathrm{pd}^{-}}\right)$independent. 
Proof. We shall prove the case $\mathcal{F}=\mathcal{F}_{\mathrm{s}}$, and the proof for the other cases is similar. Let $\left(X_{i}, T_{i}\right)$ be an $\mathcal{F}_{\mathrm{s}}$-independent t.d.s. for $i=1,2$. Let $U_{1}, \ldots, U_{n}$ and $V_{1}, \ldots, V_{n}$ be non-empty open subsets of $X_{1}$ and $X_{2}$, respectively. Then there are syndetic sets $S_{1} \in \operatorname{Ind}\left(U_{1}, \ldots, U_{n}\right)$ and $S_{2} \in \operatorname{Ind}\left(V_{1}, \ldots, V_{n}\right)$. By Lemma 5.15 there are two subsets $K_{1}, K_{2}$ of $\mathbb{Z}_{+}$such that $1_{K_{i}} \in X_{S_{i}}, i=1,2$, and $K_{1} \cap K_{2}$ is syndetic. It is clear that $K_{1} \in \operatorname{Ind}\left(U_{1}, \ldots, U_{n}\right)$ and $K_{2} \in \operatorname{Ind}\left(V_{1}, \ldots, V_{n}\right)$. Thus, $K_{1} \cap K_{2} \in$ $\operatorname{Ind}\left(U_{1} \times V_{1}, \ldots, U_{n} \times V_{n}\right)$. This implies that $\left(X_{1} \times X_{2}, T_{1} \times T_{2}\right)$ is $\mathcal{F}_{\mathrm{s}}$-independent. The theorem follows by induction.

Since a family $\mathcal{F}$ has the Ramsey property if and only if its dual family $\mathcal{F}^{*}$ has the finite intersection property, we have

Theorem 5.17. Let $\mathcal{F}$ be a family with the Ramsey property. Then the product of finitely many $\mathcal{F}^{*}$-independent t.d.s. remains $\mathcal{F}^{*}$-independent.

In [52, page 278] Weiss constructed two weakly mixing t.d.s. whose product is not transitive. (Weiss's example was only stated to be $\mathbb{Z}$-weakly mixing, but is easily checked to be $\mathbb{Z}_{+}$-weakly mixing.) In view of Theorem 5.1, this implies that the product of $\mathcal{F}_{\text {inf }}$-independent $\left(\mathcal{F}_{\text {ip }}\right.$-independent resp.) t.d.s. may fail to be $\mathcal{F}_{\text {inf-independent }}\left(\mathcal{F}_{\text {ip }}\right.$-independent resp. $)$.

\section{Classes of measurable $\mathcal{F}$-Independence}

6.1. General discussion. In this subsection we will characterize $\mathcal{F}_{\text {inf- }}$ (resp. $\mathcal{F}_{\text {ip }^{-}}, \mathcal{F}_{\text {pubd }}$ )independent m.d.s. in Theorems 6.1 and 6.2 ,

Recall that an m.d.s. $(X, T)$ is said to be ergodic if for any $A, B \in \mathcal{B}$ with positive measures, $N(A, B)$ is non-empty; it is called weakly mixing if $T \times T$ is ergodic. Similar to the topological case (Theorem 5.1) we have

Theorem 6.1. For an m.d.s. $(X, \mathcal{B}, \mu, T)$ the following are equivalent:

(1) $(X, \mathcal{B}, \mu, T)$ is weakly mixing.

(2) $(X, \mathcal{B}, \mu, T)$ is $\mathcal{F}_{\text {inf }}$-independent of order 2 .

(3) $(X, \mathcal{B}, \mu, T)$ is $\mathcal{F}_{\text {inf }}$-independent.

(4) $(X, \mathcal{B}, \mu, T)$ is $\mathcal{F}_{\text {ip }}$-independent of order 2.

(5) $(X, \mathcal{B}, \mu, T)$ is $\mathcal{F}_{\mathrm{ip}}$-independent.

Proof. It is clear that $(5) \Rightarrow(4) \Rightarrow(2)$ and $(5) \Rightarrow(3) \Rightarrow(2)$. The implication $(2) \Rightarrow(1)$ follows from the fact that if for any $A, B \in \mathcal{B}$ with positive measures one has $N(A, B) \cap N(A, A) \neq \emptyset$, then $(X, \mathcal{B}, \mu, T)$ is weakly mixing [13, Theorem 4.31]. (13, Theorem 4.31] was proved only for invertible m.d.s., as it depended on [13, Theorem 4.30] which in turn was only proved for invertible m.d.s.; 39, Theorem 3.4] proved [13, Theorem 4.30] for any m.d.s., thus [13, Theorem 4.31] also holds for any m.d.s.)

When $T$ is weakly mixing, so is $T \times \cdots \times T$ [51, Theorem 1.24]. Thus the proof of $(1) \Rightarrow(5)$ in Theorem 5.1 also applies here.

It was proved in [32, Theorem 8.3] and [35, Theorem 3.16] that a t.d.s. is topological $\mathrm{K}$ if and only if each of its finite covers by non-dense open subsets has positive entropy. Moreover, it is shown in [32, Theorem 9.4] that there exists a

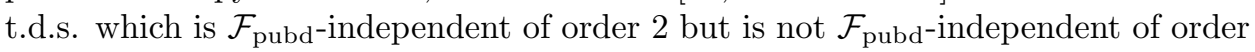
3. Now we show that in the measurable setup the situation is different. 
We refer the reader to [39, Chapter 4] for the basics of entropy theory. An m.d.s. $(X, \mathcal{B}, \mu, T)$ is said to have completely positive entropy if for every non-trivial countable measurable partition $\alpha$ of $X$ with $0<H(\alpha)<\infty$ one has $h_{\mu}(T, \alpha)>0$. The Rohlin-Sinai theorem says that an invertible m.d.s. has completely positive entropy if and only if it is a K-automorphism [43], [39, Theorem 4.12].

For $a \geq 2$ let $\Omega_{a}=\{0,1, \ldots, a-1\}^{\mathbb{Z}}$ and $Y \subseteq \Omega_{a}$. A subset $I \subseteq \mathbb{Z}$ is called an interpolating set for $Y$ if $\left.Y\right|_{I}=\left.\Omega_{a}\right|_{I}$. Now suppose that $(X, \overline{\mathcal{B}}, \mu, T)$ is an invertible m.d.s. and that $\mathcal{P}=\left\{P_{0}, P_{1}, \ldots, P_{a-1}\right\}$ is a finite measurable partition of $X$. Construct a set $Y_{\mathcal{P}} \subseteq \Omega_{a}$ as follows:

$$
Y_{\mathcal{P}}=\left\{\omega \in \Omega_{a}: \text { for all non-empty finite subsets } J \subseteq \mathbb{Z}, \mu\left(\bigcap_{j \in J} T^{-j} P_{\omega_{j}}\right)>0\right\} .
$$

Glasner and Weiss showed that an invertible m.d.s. $(X, \mathcal{B}, \mu, T)$ has completely positive entropy if and only if for every finite measurable partition $\mathcal{P}=\left\{P_{0}, P_{1}, \ldots, P_{a-1}\right\}$ of $X$ with $\min _{0 \leq j \leq a-1} \mu\left(P_{j}\right)>0$, the set $Y_{\mathcal{P}}$ has interpolating sets of positive density. In our terminology, clearly interpolating sets of $\mathcal{P}$ are exactly the independence sets of the tuple $\left(P_{0}, P_{1}, \ldots, P_{a-1}\right)$. Now we extend the result of Glasner and Weiss to general m.d.s.

Theorem 6.2. Let $(X, \mathcal{B}, \mu, T)$ be an m.d.s. Then the following are equivalent:

(1) $(X, \mathcal{B}, \mu, T)$ is $\mathcal{F}_{\text {pubd }}$-independent.

(2) $(X, \mathcal{B}, \mu, T)$ is $\mathcal{F}_{\text {pubd-independent of order } 2 .}$

(3) $(X, \mathcal{B}, \mu, T)$ has completely positive entropy.

To prove Theorem 6.2 we need some preparation. For a Lebesgue space $(X, \mathcal{B}, \mu)$ and a measurable partition $\alpha$ of $X$, we denote by $\hat{\alpha}$ the $\sigma$-algebra generated by the items of $\alpha$; for a family $\left\{\mathcal{B}_{j}\right\}_{j \in J}$ of sub- $\sigma$-algebras of $\mathcal{B}$, we denote by $\bigvee_{j \in J} \mathcal{B}_{j}$ the sub- $\sigma$-algebra of $\mathcal{B}$ generated by $\bigcup_{j \in J} \mathcal{B}_{j}$. For an m.d.s. $(X, \mathcal{B}, \mu, T)$, a measurable partition $\alpha$ of $X$, and $0 \leq n \leq m \leq \infty$, we denote $\bigvee_{j=n}^{m} T^{-j} \alpha$ and $\bigvee_{j=n}^{m} T^{-j} \hat{\alpha}$ by $\alpha_{n}^{m}$ and $\hat{\alpha}_{n}^{m}$, respectively. The following lemma is [39, Lemma 4.6] for non-invertible m.d.s.

Lemma 6.3. Let $(X, \mathcal{B}, \mu, T)$ be an m.d.s., and let $\alpha$ and $\beta$ be countable measurable partitions of $X$ with $H(\alpha), H(\beta)<\infty$. If $\beta \leq \alpha$ or $\alpha \leq \beta$, then

$$
\lim _{n \rightarrow+\infty} \frac{1}{n} H\left(\alpha_{0}^{n-1} \mid \hat{\beta}_{n}^{\infty}\right)=H\left(\alpha \mid \hat{\alpha}_{1}^{\infty}\right) .
$$

Proof. We follow the proof of [39, Lemma 4.6]. First consider the case $\beta \leq \alpha$. The sequence of $\sigma$-algebras $\left\{\hat{\alpha}_{1}^{n} \vee \hat{\beta}_{n+1}^{\infty}\right\}_{n \in \mathbb{N}}$ is increasing, and their union generates the $\sigma$-algebra $\hat{\alpha}_{1}^{\infty}$. By the increasing Martingale theorem [39, Theorem 2.6] one has

$$
\lim _{n \rightarrow+\infty} H\left(\alpha \mid \hat{\alpha}_{1}^{n} \vee \hat{\beta}_{n+1}^{\infty}\right)=H\left(\alpha \mid \hat{\alpha}_{1}^{\infty}\right)
$$

Since

$$
\begin{aligned}
H\left(\alpha_{0}^{n-1} \mid \hat{\beta}_{n}^{\infty}\right) & =\sum_{i=0}^{n-1} H\left(T^{-i} \alpha \mid \hat{\alpha}_{i+1}^{n-1} \vee \hat{\beta}_{n}^{\infty}\right)=\sum_{i=0}^{n-1} H\left(\alpha \mid \hat{\alpha}_{1}^{n-1-i} \vee \hat{\beta}_{n-i}^{\infty}\right) \\
& =\sum_{i=0}^{n-1} H\left(\alpha \mid \hat{\alpha}_{1}^{i} \vee \hat{\beta}_{i+1}^{\infty}\right),
\end{aligned}
$$

we conclude that $\lim _{n \rightarrow+\infty} \frac{1}{n} H\left(\alpha_{0}^{n-1} \mid \hat{\beta}_{n}^{\infty}\right)=H\left(\alpha \mid \hat{\alpha}_{1}^{\infty}\right)$. 
Next we consider the case $\alpha \leq \beta$. One has

$$
\limsup _{n \rightarrow+\infty} \frac{1}{n} H\left(\alpha_{0}^{n-1} \mid \hat{\beta}_{n}^{\infty}\right) \leq \lim _{n \rightarrow+\infty} \frac{1}{n} H\left(\alpha_{0}^{n-1} \mid \hat{\alpha}_{n}^{\infty}\right)=H\left(\alpha \mid \hat{\alpha}_{1}^{\infty}\right),
$$

where the second equality comes from the above paragraph. One also has

$\frac{1}{n} H\left(\beta_{0}^{n-1} \mid \hat{\beta}_{n}^{\infty}\right)=\frac{1}{n} H\left(\beta_{0}^{n-1} \vee \alpha_{0}^{n-1} \mid \hat{\beta}_{n}^{\infty}\right)=\frac{1}{n} H\left(\alpha_{0}^{n-1} \mid \hat{\beta}_{n}^{\infty}\right)+\frac{1}{n} H\left(\beta_{0}^{n-1} \mid \hat{\alpha}_{0}^{n-1} \vee \hat{\beta}_{n}^{\infty}\right)$

and

$$
\frac{1}{n} H\left(\beta_{0}^{n-1} \mid \hat{\alpha}_{n}^{\infty}\right)=\frac{1}{n} H\left(\beta_{0}^{n-1} \vee \alpha_{0}^{n-1} \mid \hat{\alpha}_{n}^{\infty}\right)=\frac{1}{n} H\left(\alpha_{0}^{n-1} \mid \hat{\alpha}_{n}^{\infty}\right)+\frac{1}{n} H\left(\beta_{0}^{n-1} \mid \hat{\alpha}_{0}^{\infty}\right) .
$$

Since $H\left(\beta_{0}^{n-1} \mid \hat{\alpha}_{0}^{n-1} \vee \hat{\beta}_{n}^{\infty}\right) \leq H\left(\beta_{0}^{n-1} \mid \hat{\alpha}_{0}^{\infty}\right)$, we get

$$
\frac{1}{n} H\left(\beta_{0}^{n-1} \mid \hat{\beta}_{n}^{\infty}\right)-\frac{1}{n} H\left(\alpha_{0}^{n-1} \mid \hat{\beta}_{n}^{\infty}\right) \leq \frac{1}{n} H\left(\beta_{0}^{n-1} \mid \hat{\alpha}_{n}^{\infty}\right)-\frac{1}{n} H\left(\alpha_{0}^{n-1} \mid \hat{\alpha}_{n}^{\infty}\right) .
$$

Taking limsup on both sides, by the above paragraph we get

$$
H\left(\beta \mid \hat{\beta}_{1}^{\infty}\right)-\liminf _{n \rightarrow+\infty} \frac{1}{n} H\left(\alpha_{0}^{n-1} \mid \hat{\beta}_{n}^{\infty}\right) \leq H\left(\beta \mid \hat{\beta}_{1}^{\infty}\right)-H\left(\alpha \mid \hat{\alpha}_{1}^{\infty}\right) .
$$

That is, $\liminf \inf _{n \rightarrow+\infty} \frac{1}{n} H\left(\alpha_{0}^{n-1} \mid \hat{\beta}_{n}^{\infty}\right) \geq H\left(\alpha \mid \hat{\alpha}_{1}^{\infty}\right)$. Therefore

$$
\lim _{n \rightarrow+\infty} \frac{1}{n} H\left(\alpha_{0}^{n-1} \mid \hat{\beta}_{n}^{\infty}\right)=H\left(\alpha \mid \hat{\alpha}_{1}^{\infty}\right),
$$

as desired.

For an m.d.s. $(X, \mathcal{B}, \mu, T)$, denote by $\mathcal{P}(T)$ the Pinsker $\sigma$-algebra of $T$ [51, page 113] consisting of $A \in \mathcal{B}$ such that $h_{\mu}(T,\{A, X \backslash A\})=0$. For a Lebesgue space $(X, \mathcal{B}, \mu)$ and sub- $\sigma$-algebras $\mathcal{B}_{1}$ and $\mathcal{B}_{2}$ of $\mathcal{B}$, we write $\mathcal{B}_{1} \subseteq_{\mu} \mathcal{B}_{2}$ if for every $A_{1} \in \mathcal{B}_{1}$ we can find $A_{2} \in \mathcal{B}_{2}$ with $\mu\left(A_{1} \Delta A_{2}\right)=0$. We also write $\mathcal{B}_{1}={ }_{\mu} \mathcal{B}_{2}$ if $\mathcal{B}_{1} \subseteq \mu \mathcal{B}_{2}$ and $\mathcal{B}_{2} \subseteq_{\mu} \mathcal{B}_{1}$. The next theorem appeared in [42, 12.3]. For the convenience of the reader, we give a proof here.

Theorem 6.4. Let $(X, \mathcal{B}, \mu, T)$ be an m.d.s. Then $\mathcal{P}(T)={ }_{\mu} \bigvee_{\alpha} \bigcap_{n \in \mathbb{Z}_{+}} \hat{\alpha}_{n}^{\infty}$ for $\alpha$ running over countable measurable partitions of $X$ with $H(\alpha)<\infty$.

Proof. Let $\alpha, \beta$ be countable measurable partitions of $X$ with $H(\alpha), H(\beta)<\infty$ and $\hat{\beta} \subseteq \bigcap_{n \in \mathbb{Z}_{+}} \hat{\alpha}_{n}^{\infty}$. For any $m \leq n$ in $\mathbb{Z}_{+}$, one has $T^{-m} \hat{\beta} \subseteq \hat{\alpha}_{n}^{\infty}$.

Thus $\frac{1}{n} H\left(\beta_{0}^{n-1} \mid \hat{\alpha}_{n}^{\infty} \vee \hat{\beta}_{n}^{\infty}\right)=0$ for every $n \in \mathbb{N}$. Taking a limit, by Lemma 6.3 we get $H\left(\beta \mid \hat{\beta}_{1}^{\infty}\right)=0$. That is, $h_{\mu}(T, \beta)=0$. Thus $\bigcap_{n \in \mathbb{Z}_{+}} \hat{\alpha}_{n}^{\infty} \subseteq \mathcal{P}(T)$.

Conversely, let $A \in \mathcal{P}(T)$. Set $\alpha=\{A, X \backslash A\}$. Then $0=h_{\mu}(T, \alpha)=$ $H\left(\alpha \mid \alpha_{1}^{\infty}\right)$. Thus $\hat{\alpha} \subseteq_{\mu} \hat{\alpha}_{1}^{\infty}$ [14, Proposition 14.18.1]. It follows that $T^{-n} \hat{\alpha} \subseteq_{\mu} \hat{\alpha}_{n+1}^{\infty}$ and hence $\hat{\alpha}_{n}^{\infty} \subseteq_{\mu} \hat{\alpha}_{n+1}^{\infty}$ for every $n \in \mathbb{Z}_{+}$. Then for each $n \in \mathbb{N}$ we can find $A_{n} \in \hat{\alpha}_{n}^{\infty}$ with $\mu\left(A \Delta A_{n}\right)=0$. Note that $\bigcap_{n \in \mathbb{N}} \bigcup_{m>n} A_{m} \in \bigcap_{n \in \mathbb{Z}_{+}} \hat{\alpha}_{n}^{\infty}$ and $\mu\left(A \Delta\left(\bigcap_{n \in \mathbb{N}} \bigcup_{m \geq n} A_{m}\right)\right)=0$. Therefore $\mathcal{P}(T) \subseteq_{\mu} \bigvee_{\alpha} \bigcap_{n \in \mathbb{Z}_{+}} \hat{\alpha}_{n}^{\infty}$ for $\alpha$ running over countable measurable partitions of $X$ with $H(\alpha)<\infty$.

The next result appeared implicitly in [42, 13.2]. For completeness, we give a proof here.

Theorem 6.5. Let $(X, \mathcal{B}, \mu, T)$ be an m.d.s. Then the following are equivalent:

(1) $(X, \mathcal{B}, \mu, T)$ has completely positive entropy. 
(2) For every countable measurable partition $\alpha$ of $X$ with $H(\alpha)<\infty$, one has $\lim _{n \rightarrow+\infty} h_{\mu}\left(T^{n}, \alpha\right)=H(\alpha)$.

Proof. $(1) \Rightarrow(2)$ : Let $\alpha$ be a countable measurable partition of $X$ with $H(\alpha)<\infty$. For each $n \in \mathbb{N}$ one has $h_{\mu}\left(T^{n}, \alpha\right)=H\left(\alpha \mid \bigvee_{j=1}^{\infty} T^{-j n} \hat{\alpha}\right) \geq H\left(\alpha \mid \hat{\alpha}_{n}^{\infty}\right)$. By the decreasing Martingale theorem [14, Theorem 14.28] we have $\lim _{n \rightarrow+\infty} H\left(\alpha \mid \hat{\alpha}_{n}^{\infty}\right)=$ $H\left(\alpha \mid \bigcap_{n \in \mathbb{Z}_{+}} \hat{\alpha}_{n}^{\infty}\right)$. Since $T$ has completely positive entropy, $\mathcal{P}(T)$ is exactly the $\sigma$ algebra of measurable subsets of $X$ with measure 0 or 1 . Thus $H\left(\alpha \mid \bigcap_{n \in \mathbb{Z}_{+}} \hat{\alpha}_{n}^{\infty}\right)=$ $H(\alpha)$ by Theorem 6.4 Therefore $\liminf _{n \rightarrow+\infty} h_{\mu}\left(T^{n}, \alpha\right) \geq H(\alpha)$. On the other hand, for each $n \in \mathbb{N}$ one has $h_{\mu}\left(T^{n}, \alpha\right) \leq H(\alpha)$. Thus $\lim _{n \rightarrow+\infty} h_{\mu}\left(T^{n}, \alpha\right)=H(\alpha)$.

$(2) \Rightarrow(1)$ : Let $\alpha$ be a countable measurable partition of $X$ with $0<H(\alpha)<\infty$. For each $n \in \mathbb{N}$ one has $h_{\mu}(T, \alpha) \geq \frac{1}{n} h_{\mu}\left(T^{n}, \alpha\right)$. Since $\lim _{n \rightarrow+\infty} h_{\mu}\left(T^{n}, \alpha\right)=$ $H(\alpha)>0$, we conclude that $h_{\mu}(T, \alpha)>0$.

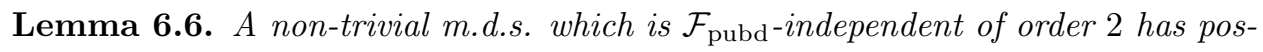
itive entropy.

Proof. Assume that $(X, \mathcal{B}, \mu, T)$ is a non-trivial m.p.s. which is $\mathcal{F}_{\text {pubd-independent }}$ of order 2 and has entropy 0 . Clearly $(X, \mathcal{B}, \mu, T)$ is ergodic. By Rosenthal's extension of the Jewett-Krieger theorem to non-invertible m.d.s. [44], there exists a t.d.s. $(\widehat{X}, \widehat{T})$ with a unique invariant Borel probability measure $\widehat{\mu}$ such that $\widehat{\mu}$ has full support and the m.d.s. $(X, \mathcal{B}, \mu, T)$ and $\left(\widehat{X}, \mathcal{B}_{\widehat{X}}, \widehat{\mu}, \widehat{T}\right)$ are isomorphic, where $\mathcal{B}_{\widehat{X}}$ denotes the Borel $\sigma$-algebra of $\widehat{X}$, in the sense that there are $X_{0} \in \mathcal{B}, \widehat{X}_{0} \in \mathcal{B}_{\widehat{X}}$ and a measure-preserving bijection $\phi: X_{0} \rightarrow \widehat{X}_{0}$ with $\mu\left(X_{0}\right)=\widehat{\mu}\left(\widehat{X}_{0}\right)=1, T X_{0} \subseteq X_{0}$, $\widehat{T} \widehat{X}_{0} \subseteq \widehat{X}_{0}$, and $\phi \circ T=\widehat{T} \circ \phi$. By the variational principle [51, Theorem 8.6], one has

$$
h_{\text {top }}(\widehat{T})=h_{\widehat{\mu}}(\widehat{T})=h_{\mu}(T)=0 \text {. }
$$

Since $(X, \mathcal{B}, \mu, T)$ is non-trivial, $(\widehat{X}, \widehat{T})$ is a non-trivial t.d.s. Thus we can find two disjoint closed subsets $A, B$ of $\widehat{X}$ with $\widehat{\mu}(A)>0, \widehat{\mu}(B)>0$. Set $\mathcal{U}=\{\widehat{X} \backslash A, \widehat{X} \backslash B\}$. Then $\mathcal{U}$ is an open cover of $\widehat{X}$, and for any $F \in \operatorname{Ind}(A, B)$ we have

$$
\begin{aligned}
0 & =h_{\text {top }}(\widehat{T}) \geq h_{\text {top }}(\widehat{T}, \mathcal{U})=\lim _{n \rightarrow+\infty} \frac{1}{n} \log N\left(\bigvee_{i=0}^{n-1} \widehat{T}^{-i} \mathcal{U}\right) \\
& \geq \limsup _{n \rightarrow+\infty} \frac{1}{n} \log N\left(\bigvee_{i \in F \cap\{0,1, \ldots, n-1\}} \widehat{T}^{-i} \mathcal{U}\right) \\
& =\limsup _{n \rightarrow+\infty} \frac{1}{n} \log 2^{|F \cap\{0,1, \ldots, n-1\}|}=\bar{d}(F) \log 2 .
\end{aligned}
$$

Hence $\bar{d}(F)=0$. Thus $\operatorname{Ind}(A, B) \cap \mathcal{F}_{\text {pud }}=\emptyset$. It follows from Example 2.2 and Proposition 3.7 that $\operatorname{Ind}(A, B) \cap \mathcal{F}_{\text {pubd }}=\emptyset$. Thus $\left(\widehat{X}, \mathcal{B}_{\widehat{X}}, \widehat{\mu}, \widehat{T}\right)$ is not $\mathcal{F}_{\text {pubd }}$ independent of order 2. Then by $\operatorname{Remark} 4.4(X, \mathcal{B}, \mu, T)$ is not $\mathcal{F}_{\text {pubd-independent }}$ of order 2 either.

We shall need the following consequence of Karpovsky and Milman's generalization of the Sauer-Perles-Shelah lemma [33, 48, 49].

Lemma 6.7 (33]). Given $r \geq 2$ in $\mathbb{N}$ and $\lambda>\ln (r-1)$ there exists a constant $c>0$ depending only on $r$ and $\lambda$ such that for all $n \in \mathbb{N}$ and $S \subseteq\{1,2, \ldots, r\}^{\{0,1, \ldots, n-1\}}$ 
satisfying $|S| \geq e^{\lambda n}$ there is an $I \subseteq\{0,1,2, \ldots, n-1\}$ with $|I| \geq$ cn and $\left.S\right|_{I}=$ $\{1,2, \ldots, r\}^{I}$.

Now we are ready to prove Theorem 6.2.

Proof of Theorem 6.2. When $(X, \mathcal{B}, \mu, T)$ is a trivial system, this is obvious. So we suppose that $(X, \mathcal{B}, \mu, T)$ is non-trivial. $(1) \Rightarrow(2)$ is obvious.

$(3) \Rightarrow(1)$ : We claim first that $(X, \mathcal{B}, \mu)$ is non-atomic in the sense that $\mu(\{x\})=0$ for every $x \in X$. In fact, since $T$ has completely positive entropy, it is ergodic. If $\mu(\{x\})>0$ for some $x \in X$, then we can find some $n \in \mathbb{N}$ such that $x, T x, \ldots, T^{n-1} x$ are pairwise distinct, $T^{n} x=x$, and $\mu(x)=\mu(T x)=\cdots=\mu\left(T^{n-1} x\right)=\frac{1}{n}$. If $n>1$, denoting by $\beta$ the partition of $X$ into $\{x\}$ and its complement, we have $h_{\mu}(T, \beta)=0$. Thus $n=1$, which means that $(X, \mathcal{B}, \mu, T)$ is trivial. Therefore $(X, \mathcal{B}, \mu)$ is non-atomic.

Given a tuple $\left(A_{1}, \ldots, A_{k}\right)$ of sets in $\mathcal{B}$ with positive measures, we are going to show that $\operatorname{Ind}\left(A_{1}, \ldots, A_{k}\right) \cap \mathcal{F}_{\text {pubd }} \neq \emptyset$. Without loss of generality, we may assume that $A_{1}, \ldots, A_{k}$ are pairwise disjoint.

Every non-atomic Lebesgue space is isomorphic to the closed unit interval endowed with its Borel $\sigma$-algebra and the Lebesgue measure [34, Theorem 17.41]. It follows that there exist an $r \in \mathbb{N}$ and a measurable partition $\alpha=\left\{B_{1}, \ldots, B_{r}\right\}$ of $X$ such that $r>k, \mu\left(B_{i}\right)=\frac{1}{r}$ for $i=1,2, \ldots, r$, and $B_{j}$ is a subset of $A_{j}$ for $j=1,2, \ldots, k$. To show $\operatorname{Ind}\left(A_{1}, \ldots, A_{k}\right) \cap \mathcal{F}_{\text {pubd }} \neq \emptyset$, it is sufficient to show $\operatorname{Ind}\left(B_{1}, \ldots, B_{r}\right) \cap \mathcal{F}_{\text {pubd }} \neq \emptyset$.

By Theorem 6.5 we have $\lim _{n \rightarrow+\infty} h_{\mu}\left(T^{n}, \alpha\right)=H(\alpha)=\ln r$. Thus there exists $\ell \in \mathbb{N}$ such that $\lambda:=h_{\mu}\left(T^{\ell}, \alpha\right)>\ln (r-1)$. Then $\frac{1}{n} H\left(\bigvee_{i=0}^{n-1} T^{-\ell i} \alpha\right) \geq \lambda>\ln (r-1)$ for all $n \in \mathbb{N}$. For any given finite measurable partition $\beta$ of $X$, we define

$$
|\beta|_{\mu}=|\{B \in \beta: \mu(\beta)>0\}| \text {. }
$$

Then $\left|\bigvee_{i=0}^{n-1} T^{-\ell i} \alpha\right|_{\mu} \geq e^{H\left(\bigvee_{i=0}^{n-1} T^{-\ell i} \alpha\right)} \geq e^{\lambda n}$ for all $n \in \mathbb{N}$.

Now combing this with Lemma 6.7 we see that there exists a constant $c>0$ depending on only $r$ and $\lambda$ such that for any $n \in \mathbb{N}$ there is an $I_{n} \subseteq\{0,1,2, \ldots, n-1\}$ with $\left|I_{n}\right| \geq c n$ and $\mu\left(\bigcap_{i \in I_{n}} T^{-\ell i} B_{s(i)}\right)>0$ for any $s \in\{1,2, \ldots, r\}^{I_{n}}$. This implies that $\ell I_{n} \in \operatorname{Ind}\left(B_{1}, \ldots, B_{r}\right)$ for each $n \in \mathbb{N}$. From Lemma 4.5 we conclude that $\operatorname{Ind}\left(B_{1}, \ldots, B_{r}\right) \cap \mathcal{F}_{\mathrm{pd}} \neq \emptyset$.

$(2) \Rightarrow(3)$ : Assume that $(X, \mathcal{B}, \mu, T)$ is $\mathcal{F}_{\text {pubd-independent of order } 2 \text {. Note that }}$ the definitions of independence sets and entropy apply to more general measuretheoretical dynamical systems in which the probability space does not have to be a Lebesgue space. In this sense $(X, \mathcal{P}(T), \mu, T)$ is also $\mathcal{F}_{\text {pubd-independent of }}$ order 2 and has entropy 0 . Since $(X, \mathcal{B}, \mu)$ is a Lebesgue space, it is easy to see that $\mathcal{B}$ is separable under the semi-metric $d(A, B)=\mu(A \Delta B)$. Then $\mathcal{P}(T)$ is also separable under this semi-metric. It follows that there is an m.d.s. $(Y, \mathcal{J}, \nu, S)$ (i.e., $(Y, \mathcal{J}, \nu)$ is a Lebesgue space) such that the measure algebra triples associated to $(X, \mathcal{P}(T), \mu, T)$ and $(Y, \mathcal{J}, \nu, S)$ in Remark 4.4 are isomorphic [13, Proposition 5.3]. Then $(Y, \mathcal{J}, \nu, S)$ is also $\mathcal{F}_{\text {pubd }}$-independent of order 2 and has entropy 0 . By Lemma 6.6 $(Y, \mathcal{J}, \nu, S)$ is trivial. Thus $\mathcal{P}(T)$ consists of measurable subsets of $X$ with measure 0 or 1 . That is, $(X, \mathcal{B}, \mu, T)$ has completely positive entropy.

6.2. Non-existence of $\mathcal{F}_{\mathrm{s}}$-independent m.d.s. In this subsection we establish the somewhat surprising result that there is no non-trivial m.d.s. which is $\mathcal{F}_{\mathrm{s}^{-}}$ independent. In the following, we aim to show that for any non-periodic m.d.s. 
$(X, \mathcal{B}, \mu, T)$ there exists $A \in \mathcal{B}$ with $\mu(A)>0$ such that $\operatorname{Ind}(A)$ does not contain a syndetic set.

An m.d.s. $(X, \mathcal{B}, \mu, T)$ is called non-periodic or free if $\mu\left(\left\{x \in X: T^{n} x=x\right\}\right)=0$ for every $n \in \mathbb{N}$. It is easy to see that an ergodic m.d.s. $(X, \mathcal{B}, \mu, T)$ is non-periodic if and only if $(X, \mathcal{B}, \mu)$ is non-atomic in the sense that $\mu(x)=0$ for every $x \in X$.

Theorem 6.8. Let $(X, \mathcal{B}, \mu, T)$ be a non-periodic m.d.s. Then for any $\varepsilon>0$ there exists $A \in \mathcal{B}$ with $\mu(A)>1-\varepsilon$ such that $\operatorname{Ind}(A)$ does not contain any syndetic set.

Proof. Endow $X$ with a Polish topology such that $\mathcal{B}$ is the corresponding Borel $\sigma$-algebra. Replacing the Polish topology on $X$ by a finer one if necessary [34, Theorem 13.11 and Lemma 13.3], we may assume that $T$ is continuous. Let $\varepsilon>0$. We claim that there is a compact subset $K$ of $X$ such that $\mu(K)>1-\varepsilon$ and $A_{n}:=\bigcap_{j \in \mathbb{Z}_{+}} \bigcup_{i=0}^{n-1} T^{-j-i} K$ has measure 0 for every $n \in \mathbb{N}$. Assuming this claim let us show how it implies the theorem.

Since $\mu\left(\bigcup_{n \in \mathbb{N}} A_{n}\right)=0$, one has $\mu\left(K \backslash\left(\bigcup_{n \in \mathbb{N}} A_{n}\right)\right)=\mu(K)>1-\varepsilon$. By the regularity of $\mu$ [34, Theorem 17.11], we can find a compact set $A$ contained in $K \backslash\left(\bigcup_{n \in \mathbb{N}} A_{n}\right)$ such that $\mu(A)>1-\varepsilon$. We shall show that $\operatorname{Ind}(A)$ does not contain any syndetic set.

Let $F \in \operatorname{Ind}(A)$ be non-empty. Replacing $F$ by $F-\min F$ if necessary, we may assume that $0 \in F$. One has $\mu\left(\bigcap_{j \in J} T^{-j} A\right)>0$ and hence $\bigcap_{j \in J} T^{-j} A \neq \emptyset$ for every non-empty finite subset $J$ of $F$. Since $A$ is compact, we conclude that $\bigcap_{j \in F} T^{-j} A$ is non-empty. Take $x \in \bigcap_{j \in F} T^{-j} A$. Then $x \in A$ and $T^{j} x \in A \subseteq K$ for every $j \in F$. For each $n \in \mathbb{N}$ one has $x \notin A_{n}$, and hence for some $j_{n} \in \mathbb{Z}_{+}$none of $T^{j_{n}} x, T^{j_{n}+1} x, \ldots, T^{j_{n}+n-1} x$ is in $K$. Then $\left[j_{n}, j_{n}+n-1\right] \cap F=\emptyset$. Therefore $F$ is not syndetic.

We are left to prove the above claim. Since the main idea of the proof is well illustrated in the case where $\mu$ is ergodic, we consider this case first.

So assume that $\mu$ is ergodic. Since $(X, \mathcal{B}, \mu, T)$ is non-periodic, by the comment before Theorem $6.8(X, \mathcal{B}, \mu)$ is non-atomic. Replacing $X$ by $\operatorname{supp}(\mu)$ if necessary, we may assume that $\mu$ has full support. Take $x \in X$ and set $W=\left\{T^{n} x: n \in \mathbb{Z}_{+}\right\}$. Then $T W \subseteq W$, and $W$ is non-empty and countable. Since $\mu$ is non-atomic, one has $\mu(W)=0$, and hence $\mu(X \backslash W)=1$. By the regularity of $\mu$, we can find a compact set $K$ contained in $X \backslash W$ such that $\mu(K)>1-\varepsilon$. For any $n \in \mathbb{N}, \bigcup_{i=0}^{n} T^{-i} K$ is a closed subset of $X$ with $\bigcup_{i=0}^{n} T^{-i} K \neq X$, since $W \cap\left(\bigcup_{i=0}^{n} T^{-i} K\right)=\emptyset$. As $\mu$ has full support, $\mu\left(\bigcup_{i=0}^{n} T^{-i} K\right)<1$ for all $n \in \mathbb{N}$. Note that $A_{n} \in \mathcal{B}$ and $T^{-1} A_{n} \supseteq A_{n}$. Since $\mu$ is ergodic and $\mu\left(A_{n}\right) \leq \mu\left(\bigcup_{i=0}^{n} T^{-i} K\right)<1$, we get $\mu\left(A_{n}\right)=0$. This finishes the proof in the case where $\mu$ is ergodic.

Now we consider the general case, using the ergodic decomposition of $(X, \mathcal{B}, \mu, T)$.

Denote by $P(X)$ the set of all probability Borel measures on $X$, and endow it with the $\sigma$-algebra generated by the functions $\mu^{\prime} \mapsto \mu^{\prime}(A)$ on $P(X)$ for all $A \in \mathcal{B}$ [34, Section 17.E].

From the ergodic decomposition of $(X, \mathcal{B}, \mu, T)$ we know that there exist a set $X^{\prime} \in \mathcal{B}$ with $\mu\left(X^{\prime}\right)=1$ and $T X^{\prime} \subseteq X^{\prime}$, a Lebesgue space $(Y, \mathcal{J}, \nu)$, a measurable map $\pi: X^{\prime} \rightarrow Y$, a measurable map $y \mapsto \mu_{y}$ from $Y$ to $P(X)$, and a set $Y^{\prime} \in \mathcal{J}$ with $\nu\left(Y^{\prime}\right)=1$ such that $\pi T=\pi, \pi \mu=\nu, \mu(A)=\int_{Y} \mu_{y}(A) d \nu(y)$ for all $A \in \mathcal{B}$, and $\mu_{y}\left(\pi^{-1}(y)\right)=1$ and $T \mu_{y}=\mu_{y}$ and $\left(X, \mathcal{B}, \mu_{y}, T\right)$ is ergodic for every $y \in Y^{\prime}$ [14, Theorem 3.42]. ([14, Theorem 3.42] was only proved for invertible m.d.s., but it is easy to see that the proof works for any m.d.s.) 
Set $W_{1}=\left\{x \in X: T^{n} x=x\right.$ for some $\left.n \in \mathbb{N}\right\}$. Clearly $W_{1}$ is in $\mathcal{B}$. By assumption $0=\mu\left(W_{1}\right)=\int_{Y} \mu_{y}\left(W_{1}\right) d \nu(y)$. Thus $\mu_{y}\left(W_{1}\right)=0$ for $\nu$ a.e. $y \in$ $Y$. Replacing $Y^{\prime}$ by a smaller measurable set if necessary, we may assume that $\mu_{y}\left(W_{1}\right)=0$ for every $y \in Y^{\prime}$. Since $\left(X, \mathcal{B}, \mu_{y}, T\right)$ is ergodic for every $y \in Y^{\prime}$, it follows that $\mu_{y}$ is non-atomic for every $y \in Y^{\prime}$.

Endow $Y$ with a Polish topology such that $\mathcal{J}$ is the corresponding Borel $\sigma$ algebra. Replacing the Polish topology on $X$ by a finer one if necessary, we may assume that $\pi$ is continuous.

Denote by $F(X)$ the set of all closed subsets of $X$, and endow it with the Effros Borel structure, i.e., the $\sigma$-algebra generated by the sets $\{Z \in F(X): Z \cap U \neq \emptyset\}$ for all open subsets $U$ of $X$. The map $\phi: P(X) \rightarrow F(X)$ sending each $\mu^{\prime}$ to $\operatorname{supp}\left(\mu^{\prime}\right)$ is measurable [34, Exercise 17.38]. By the Kuratowski-Ryll-Nardzewski selection theorem [34, Theorem 12.13] we can find a measurable map $\psi: F(X) \rightarrow X$ such that $\psi(Z) \in Z$ for each non-empty $Z \in F(X)$.

Note that $\operatorname{supp}\left(\mu_{y}\right) \subseteq \pi^{-1}(y)$ for every $y \in Y^{\prime}$. Thus the map $\varphi_{n}: Y^{\prime} \rightarrow X$ sending $y$ to $T^{n}\left(\psi\left(\phi\left(\mu_{y}\right)\right)\right)$ is measurable and injective for each $n \in \mathbb{Z}_{+}$. Recall that a measurable space is a standard Borel space if the $\sigma$-algebra is the Borel $\sigma$-algebra for some Polish topology on the set. A measurable subset of a standard Borel space together with the restriction of the $\sigma$-algebra to the subset is also a standard Borel space [34, Corollary 13.4]. Thus $Y^{\prime}$ together with the restriction of $\mathcal{J}$ on $Y^{\prime}$ is a standard Borel space. The Lusin-Souslin theorem says that the image of any injective measurable map from a standard Borel space to another standard Borel space is measurable [34, Corollary 15.2]. Thus the set $W:=\bigcup_{n \in \mathbb{Z}_{+}} \varphi_{n}\left(Y^{\prime}\right)$ is in $\mathcal{B}$. Note that $T W \subseteq W$, and $W \cap \operatorname{supp}\left(\mu_{y}\right)$ is non-empty and countable for every $y \in Y^{\prime}$.

Since $\mu_{y}$ is non-atomic for every $y \in Y^{\prime}$, one has $\mu_{y}(W)=0$ for every $y \in Y^{\prime}$. Thus $\mu(W)=\int_{Y} \mu_{y}(W) d \nu(y)=0$, and hence $\mu(X \backslash W)=1$. By the regularity of $\mu$, we can find a compact set $K$ contained in $X \backslash W$ such that $\mu(K)>1-\varepsilon$. For any $n \in \mathbb{N}$ and $y \in Y^{\prime}, \operatorname{supp}\left(\mu_{y}\right) \cap\left(\bigcup_{i=0}^{n} T^{-i} K\right)$ is a closed subset of $\operatorname{supp}\left(\mu_{y}\right)$ with $\operatorname{supp}\left(\mu_{y}\right) \cap\left(\bigcup_{i=0}^{n} T^{-i} K\right) \neq \operatorname{supp}\left(\mu_{y}\right)$, since $W \cap\left(\bigcup_{i=0}^{n} T^{-i} K\right)=\emptyset$ and $W \cap$ $\operatorname{supp}\left(\mu_{y}\right) \neq \emptyset$. Thus $\mu_{y}\left(\bigcup_{i=0}^{n} T^{-i} K\right)<1$ for all $n \in \mathbb{N}$ and $y \in Y^{\prime}$.

We still have $A_{n} \in \mathcal{B}$ and $T^{-1} A_{n} \supseteq A_{n}$. For each $y \in Y^{\prime}$, since $\left(X, \mathcal{B}, \mu_{y}, T\right)$ is ergodic, $\mu_{y}\left(A_{n}\right)$ is equal to either 0 or 1 . By the above paragraph we have $\mu_{y}\left(A_{n}\right) \leq \mu_{y}\left(\bigcup_{i=0}^{n-1} T^{-i} K\right)<1$ for each $y \in Y^{\prime}$. Thus $\mu_{y}\left(A_{n}\right)=0$ for each $y \in Y^{\prime}$. Therefore $\mu\left(A_{n}\right)=\int_{Y} \mu_{y}\left(A_{n}\right) d \nu(y)=0$, as desired. This proves the claim and finishes the proof of the theorem.

Now we are able to show

Theorem 6.9. There is no non-trivial m.d.s. which is $\mathcal{F}_{\mathrm{s}}$-independent of order 2 .

Proof. Assume to the contrary that there exists such a system $(X, \mathcal{B}, \mu, T)$. By Theorem 6.1, $T$ is weakly mixing.

By Theorem 6.8, $T$ is a.e. periodic. Then the set $A_{n}=\left\{x \in X: T^{n} x=x, T^{j} x \neq\right.$ $x$ for all $1 \leq j<n\}$ has positive measure for some $n \in \mathbb{N}$. Note that $T A_{n}=A_{n}$. By [24, page 70] we can find $B \subseteq A_{n}$ such that $B \in \mathcal{B}, \mu(B)=\mu\left(A_{n}\right) / n$, and $B, T B, \ldots, T^{n-1} B$ are pairwise disjoint. If $n \geq 2$, then $N(B, B) \cap N(B, T B)=\emptyset$, which contradicts that $(X, \mathcal{B}, \mu, T)$ is weakly mixing. Thus $\mu\left(A_{n}\right)=0$ for every $n \geq 2$. Then $\mu\left(A_{1}\right)=1$. Since $(X, \mathcal{B}, \mu, T)$ is non-trivial, we can find some $B \subseteq A_{1}$ 
such that $B \in \mathcal{B}$ and $0<\mu(B)<1$. Then $N(B, X \backslash B)=\emptyset$, again contradicting the fact that $(X, \mathcal{B}, \mu, T)$ is weakly mixing.

Remark 6.10. Using Theorem 5.11 one can strengthen Theorem 6.9 as follows. For any non-trivial ergodic m.d.s. $(X, \mathcal{B}, \mu, T)$, Rosenthal's extension of the JewettKrieger theorem to non-invertible m.d.s. [44] says that there exists a t.d.s. $(\widehat{X}, \widehat{T})$ with a unique invariant Borel probability measure $\widehat{\mu}$ such that $\widehat{\mu}$ has full support and the m.d.s. $(X, \mathcal{B}, \mu, T)$ and $\left(\widehat{X}, \mathcal{B}_{\widehat{X}}, \widehat{\mu}, \widehat{T}\right)$ are isomorphic, where $\mathcal{B}_{\widehat{X}}$ denotes the Borel $\sigma$-algebra of $\widehat{X}$. Then $(\widehat{X}, \widehat{T})$ is minimal (see for example [51, Theorem 6.17]). Furthermore, the proof in 44 shows that we can choose $\widehat{X}$ to be a Cantor set. For any real-valued continuous function $f$ on $X$, the sequence $\left\{\frac{1}{n+1} \sum_{i=0}^{n} f \circ \widehat{T}^{i}\right\}_{n \in \mathbb{Z}_{+}}$of functions on $X$ converges to the constant function $\int_{\widehat{X}} f(x) d \widehat{\mu}(x)$ uniformly as $n \rightarrow$ $+\infty$ [51, Theorem 6.19]. By Theorem [5.11 we can find disjoint non-empty clopen subsets $\widehat{V}_{0}$ and $\widehat{V}_{1}$ of $\widehat{X}$ such that $\operatorname{Ind}\left(\widehat{V}_{0}, \widehat{V}_{1}\right) \cap \mathcal{F}_{\mathrm{s}}=\emptyset$. Say that $\widehat{V}_{j}$ corresponds to $V_{j} \in \mathcal{B}$ for $j=1,2$. Then $V_{0}$ and $V_{1}$ are disjoint and have positive measures, and $\operatorname{Ind}\left(V_{0}, V_{1}\right) \cap \mathcal{F}_{\mathrm{s}}=\emptyset$. Furthermore, taking $f$ to be $1_{\widehat{V}_{j}}$, we see that the sequence $\left\{\frac{1}{n+1} \sum_{i=0}^{n} 1_{V_{j}} \circ T^{i}\right\}_{n \in \mathbb{Z}_{+}}$converges to $\mu\left(V_{j}\right)$ in $L^{\infty}(X, \mu)$ for $j=1,2$.

6.3. Finite product. By contrast to the topological case, it is well known that the product of two weakly mixing m.d.s. is still weakly mixing 13, Proposition 4.6]. In view of Theorem 6.1, this means that the products of finitely many $\mathcal{F}_{\text {inf }}{ }^{-}$ independent $\left(\mathcal{F}_{\mathrm{ip}}\right.$-independent resp. $)$ m.d.s. are $\mathcal{F}_{\text {inf }}$-independent $\left(\mathcal{F}_{\text {ip }}\right.$-independent resp.).

Meanwhile, it is known that the product of finitely many invertible completely positive entropy m.d.s. has completely positive entropy [39, Theorem 4.14]. As the topological case, every m.d.s. has a natural extension [10, Page 240], which is always invertible. The natural extension of a completely positive entropy m.d.s. has completely positive entropy [42, 13.8] (one can also deduce this from Theorem 6.5 and the fact that the natural extension of an m.d.s. is the inverse limit of a sequence of m.d.s. identical to the original one). It follows that the product of finitely many completely positive entropy m.d.s. has completely positive entropy. In view of Theorem 6.2, this means that the product of finitely many $\mathcal{F}_{\text {pd }}$-independent m.d.s. remains $\mathcal{F}_{\mathrm{pd}}$-independent. Thus we make the following conjecture.

Conjecture 6.11. For any family $\mathcal{F}$, the product of finitely many $\mathcal{F}$-independent m.d.s. remains $\mathcal{F}$-independent.

\section{A topological proof of minimal topological K-Systems ARE STRONGLY MIXING}

In this section we prove Theorem 7.1 and Corollary 7.3

For a cover $\mathcal{V}$ of a compact space $X$ by open subsets, we denote by $N(\mathcal{V})$ the minimal cardinality of subcovers of $\mathcal{V}$. Let $\mathcal{F}$ be a family. A t.d.s. $(X, T)$ is called $\mathcal{F}$-scattering if for each $F=\left\{a_{1}<a_{2}<\cdots\right\} \in \mathcal{F}$ and each finite cover $\mathcal{U}$ of $X$ by non-dense open subsets, one has $\lim _{n \rightarrow+\infty} N\left(\bigvee_{i=1}^{n} T^{-a_{i}} \mathcal{U}\right)=\infty$. It was shown in [29, Theorem 5.5] by using ergodic theory that topological K-systems are $\mathcal{F}_{\text {inf- }}{ }^{-}$ scattering. Combining this with the fact that a minimal $\mathcal{F}_{\text {inf-scattering t.d.s. is }}$ strongly mixing [30, Theorem 5.6], one knows that a minimal topological K-system is strongly mixing [29, Theorem 5.10]. Now we give a topological proof of the fact that a topological K-system is $\mathcal{F}_{\text {inf-scattering. }}$ 
Recall that for any $F=\left\{a_{1}<a_{2}<\cdots\right\} \in \mathcal{F}_{\text {inf }}$ and any open cover $\mathcal{U}$ of $X$, the topological sequence entropy of $T$ with respect to $F$ is defined as

$$
h_{\text {top }}^{F}(T, \mathcal{U})=\limsup _{n \rightarrow+\infty} \frac{\log N\left(\bigvee_{i=1}^{n} T^{-a_{i}} \mathcal{U}\right)}{n} .
$$

Theorem 7.1. Let $(X, T)$ be a t.d.s., $n \geq 2,\left(x_{1}, \ldots, x_{n}\right)$ be an $\mathcal{F}_{\text {pubd-independent }}$ tuple of $X$ with points pairwise distinct, and $U_{1}, \ldots, U_{n}$ be pairwise disjoint closed neighborhoods of $x_{1}, \ldots, x_{n}$, respectively. Set $\mathcal{U}=\left\{U_{1}^{c}, \ldots, U_{n}^{c}\right\}$. Then for any $F \in \mathcal{F}_{\text {inf }}$, one has $h_{\text {top }}^{F}(T, \mathcal{U})>0$. Consequently, a topological $K$-system is $\mathcal{F}_{\text {inf }}$ scattering.

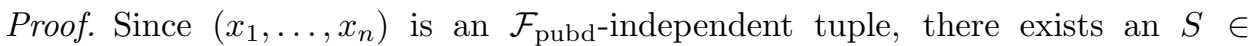
$\operatorname{Ind}\left(U_{1}, \ldots, U_{n}\right)$ with positive upper Banach density $d$. Let $F=\left\{a_{1}<a_{2}<\cdots\right\}$ in $\mathcal{F}_{\text {inf }}$. Then by Lemma 5.14 for any $k \in \mathbb{N}$, setting $q_{k}$ to be the smallest integer no less than $2 k / d$, we can find $p_{k} \in \mathbb{Z}$ and $W_{k} \subseteq\left\{a_{1}, a_{2}, \ldots, a_{q_{k}}\right\}$ with $\left|W_{k}\right|=k$ and $p_{k}+W_{k} \subseteq S$. Thus, $W_{k} \in \operatorname{Ind}\left(U_{1}, \ldots, U_{n}\right)$. This implies that

$$
\begin{aligned}
h_{\text {top }}^{F}(T, \mathcal{U}) & \geq \limsup _{k \rightarrow+\infty} \frac{1}{q_{k}} \log N\left(\bigvee_{j \in W_{k}} T^{-j} \mathcal{U}\right) \geq \limsup _{k \rightarrow+\infty} \frac{1}{q_{k}} \log \left(\frac{n}{n-1}\right)^{k} \\
& =\frac{2}{d} \log \frac{n}{n-1}>0 .
\end{aligned}
$$

Now for any finite open cover $\mathcal{V}$ of $X$ by non-dense open subsets, we may find some $n \geq 2$, pairwise distinct $x_{1}, \ldots, x_{n}$ in $X$ and pairwise disjoint closed neighborhoods $U_{1}, \ldots, U_{n}$ of $x_{1}, \ldots, x_{n}$ respectively such that $\mathcal{V}$ refines $\mathcal{U}=\left\{U_{1}^{c}, \ldots, U_{n}^{c}\right\}$. If $(X, T)$ is topological $\mathrm{K}$, then each tuple in $X$ is $\mathcal{F}_{\text {pubd-independent. Thus for }}$ any $F=\left\{a_{1}<a_{2}<\cdots\right\}$ in $\mathcal{F}_{\text {inf }}$, by the above paragraph we have $h_{\text {top }}^{F}(T, \mathcal{V}) \geq$ $h_{\text {top }}^{F}(T, \mathcal{U})>0$. This implies that $N\left(\bigvee_{i=1}^{m} T^{-a_{i}} \mathcal{V}\right) \rightarrow \infty$ as $m \rightarrow+\infty$, i.e., $(X, T)$ is $\mathcal{F}_{\text {inf-scattering. }}$

Let $\mathcal{F}$ be a family. A t.d.s. $(X, T)$ is called $\mathcal{F}$-transitive if for any non-empty open subsets $U$ and $V$ of $X$, one has $N(U, V) \in \mathcal{F}$. It is called mildly mixing if its product with any transitive t.d.s. is transitive. It was shown in 32, Theorem 7.5] that a u.p.e. system is mildly mixing. From [32, Theorem 7.3] or [35, Theorem 3.16] one knows that a t.d.s. is u.p.e. if and only if it is $\mathcal{F}_{\text {pubd-independent of order }}$ 2. Denote by $\Delta$ the family in $\mathbb{Z}_{+}$generated by the sets $F-F:=\{a-b: a, b \in$ $F, a-b>0\}$ for all $F \in \mathcal{F}_{\text {inf }}$. By [30, Theorem 6.6] every $\Delta^{*}$-transitive system is mildly mixing. Now we strengthen the above result to show that every t.d.s.

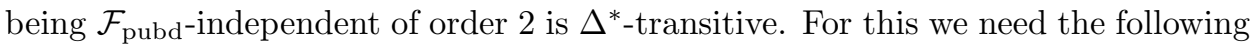
proposition, which appeared in [13, page 84] (see also [53, Proposition 2.3]) and also follows directly from Lemma 5.14

Proposition 7.2. If $F \in \mathcal{F}_{\text {pubd, }}$, then $F-F$ is in $\Delta^{*}$.

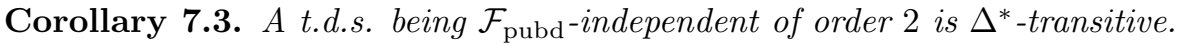

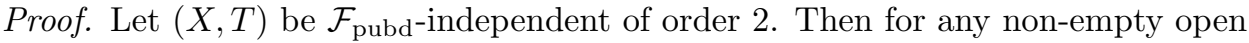
subsets $U$ and $V$ of $X$, there exists an $F \in \operatorname{Ind}(U, V) \cap \mathcal{F}_{\text {pubd. }}$. Clearly $F-F \subseteq$ $N(U, V)$. By Proposition 7.2 one has $N(U, V) \in \Delta^{*}$.

To end this section we make the following remark. Denote by $\mathcal{F}_{\text {ss }}$ the family consisting of $S \subseteq \mathbb{Z}_{+}$satisfying that for each $F \in \mathcal{F}_{\text {inf }}$ and each $k \in \mathbb{N}$ there exists $p_{k} \in \mathbb{Z}$ with $\left|F \cap\left(S+p_{k}\right)\right| \geq k$. It is clear that for any $S \in \mathcal{F}_{\text {ss }}$ one has $S-S \in \Delta^{*}$. 
Remark 7.4. One obvious corollary of Lemma 5.14 is that $\mathcal{F}_{\text {pubd }} \subseteq \mathcal{F}_{\text {ss }}$. We remark that there exists an $S \in \mathcal{F}_{\text {ss }}$ containing no arithmetic progression of length 3 (and thus having zero upper Banach density by Roth's theorem [47]).

Proof. For $k \geq 3$ set

$S_{k}=\left\{\left\{a_{1}, a_{2}, \ldots, a_{k}\right\} \subseteq \mathbb{N}: a_{j}-a_{i}>a_{i}-a_{s}>0\right.$ for all $\left.1 \leq s<i<j \leq k\right\}$.

Each $S_{k}$ is countable. Enumerate $\bigcup_{k>3} S_{k}$ as $\left\{A_{1}, A_{2}, \ldots\right\}$. Now let $\left\{t_{i}\right\}_{i \in \mathbb{N}}$ be a sequence in $\mathbb{N}$ and set $S=\bigcup_{i \in \mathbb{N}}\left(A_{i}+\bar{t}_{i}\right)$.

Now assume that $F$ is an infinite subset of $\mathbb{Z}$. For each $k \geq 3$, inductively we can find $b_{1}, b_{2}, \ldots, b_{k} \in F$ such that $b_{j}-b_{i}>b_{i}-b_{s}>0$ for all $1 \leq s<i<j \leq k$. This implies that there exists $p_{k} \in \mathbb{Z}$ with $\left|F \cap\left(S+p_{k}\right)\right| \geq k$. Thus $S$ is in $\mathcal{F}_{\text {ss }}$.

If we choose $t_{i}$ to grow rapidly enough, it is easy to check that $S$ does not contain any arithmetic progression of length 3 .

We remark that the set of prime numbers is not in $\mathcal{F}_{\mathrm{ss}}$. In fact any $S=\left\{a_{1}<\right.$ $\left.a_{2}<\cdots\right\} \in \mathcal{F}_{\text {inf }}$ with $a_{i+1}-a_{i} \rightarrow+\infty$ as $i \rightarrow+\infty$ is not in $\mathcal{F}_{\text {ss }}$. Actually one can find an $F \in \mathcal{F}_{\text {inf }}$ such that $|F \cap(S+p)| \leq 2$ for all $p \in \mathbb{Z}$. To find such an $F$, start with any $b_{1}<b_{2}$ in $\mathbb{N}$. Since $a_{i+1}-a_{i} \rightarrow+\infty$ as $i \rightarrow+\infty$, there are only finitely many $i$ and $j$ satisfying $a_{j}-a_{i}=b_{2}-b_{1}$. From this we can find $b_{3}>b_{2}$ in $\mathbb{N}$ such that $\left|\left\{b_{1}, b_{2}, b_{3}\right\} \cap(S+p)\right| \leq 2$ for every $p \in \mathbb{Z}$. Inductively we find $b_{3}<b_{4}<b_{5}<\cdots$ in $\mathbb{N}$ such that $\left|\left\{b_{1}, b_{2}, \ldots, b_{k}\right\} \cap(S+p)\right| \leq 2$ for every $k \in \mathbb{N}$ and $p \in \mathbb{Z}$.

\section{ApPEndix}

In this appendix we prove Theorem 5.12

Proof of Theorem 5.12. Take $d \in \mathbb{N}$ with $p^{d}>\frac{(d+p) \ell}{p}$. Let $m>d$ be large enough, which we shall determine later. It suffices to show that for every $n \in \mathbb{N}$ there exists $x_{n} \in \Sigma_{p}$ with $x_{n}[j, j+m-1] \notin A_{j}$ for all $0 \leq j \leq n$. Then any limit point of the sequence $\left\{x_{n}\right\}_{n \in \mathbb{Z}_{+}}$in $\Sigma_{p}$ satisfies the requirement.

As convention, set $\Lambda_{p}^{0}$ to be the one element set consisting of the empty word. For any $k, s, t \in \mathbb{Z}_{+}$and any $y \in \Lambda_{p}^{k}$, set $|y|=k$ and denote by $\Lambda_{p}^{s} y \Lambda_{p}^{t}$ the subset of $\Lambda_{p}^{s+k+t}$ consisting of elements of the form $w y z$ for some $w \in \Lambda_{p}^{s}$ and $z \in \Lambda_{p}^{t}$.

For each $n \in \mathbb{Z}_{+}$set $B_{n}$ to be the subset of $\Lambda_{p}^{m}$ consisting of elements $b$ for which there is no $x \in \Sigma_{p}$ with $x[n, n+m-1]=b$ and $x[j, j+m-1] \notin A_{j}$ for all $0 \leq j \leq n$. Set $C_{n}$ to be the subset of $\Lambda_{p}^{m-1}$ consisting of elements $c$ for which $\Lambda_{p} c$ is contained in $B_{n}$. Note that $C_{n}$ is exactly the set of elements $c \in \Lambda_{p}^{m-1}$ for which there is no $x \in \Sigma_{p}$ with $x[n+1, n+m-1]=c$ and $x[j, j+m-1] \notin A_{j}$ for all $0 \leq j \leq n$. It follows that

$$
B_{n+1}=A_{n+1} \cup\left(\bigcup_{c \in C_{n}} c \Lambda_{p}\right)
$$

Then

$$
p\left|C_{n+1}\right| \leq\left|B_{n+1}\right|=\left|A_{n+1} \cup\left(\bigcup_{c \in C_{n}} c \Lambda_{p}\right)\right| \leq\left|A_{n+1}\right|+p\left|C_{n}\right| \leq \ell+p\left|C_{n}\right|
$$

for all $n \in \mathbb{Z}_{+}$. Clearly $\left|C_{0}\right| \leq \frac{\left|B_{0}\right|}{p} \leq \frac{\ell}{p}$. Inductively one gets that $\left|C_{n}\right| \leq \frac{(n+1) \ell}{p}$ for all $n \in \mathbb{Z}_{+}$. 
Set $D_{n}$ to be the subset of $\bigcup_{k=0}^{m-1} \Lambda_{p}^{k}$ consisting of elements $y$ such that $y \Lambda_{p}^{m-1-|y|}$ $\subseteq C_{n}$ but $y[1,|y|-1] \Lambda_{p}^{m-|y|} \nsubseteq C_{n}$. We put $\Lambda_{p}^{0} \subseteq D_{n}$ exactly when $C_{n}=\Lambda_{p}^{m-1}$. Note that $C_{n}$ is the disjoint union of $y \Lambda_{p}^{m-1-|y|}$ for $y \in D_{n}$. For each $0 \leq k \leq m-1$ set $D_{n, k}=\left\{y \in D_{n}:|y|=k\right\}$. We claim that

$$
\left|D_{n+1, k}\right| \leq \ell+\sum_{j=k+1}^{m-1}\left|D_{n, j}\right|
$$

for all $n \in \mathbb{Z}_{+}$and $0 \leq k \leq m-1$. This is clearly true if $k=0$ or $D_{n, 0} \neq$ $\emptyset$. Thus assume that $1 \leq k \leq m-1$ and $D_{n, 0}=\emptyset$. Let $y \in D_{n+1, k}$. Then $y \Lambda_{p}^{m-1-k}$ is contained in $C_{n+1}$, and hence $\Lambda_{p} y \Lambda_{p}^{m-1-k}$ is contained in $B_{n+1}=$ $A_{n+1} \cup\left(\bigcup_{z \in D_{n}} z \Lambda_{p}^{m-|z|}\right)$. If $\Lambda_{p} y \Lambda_{p}^{m-1-k}$ has non-empty intersection with $z_{j} \Lambda_{p}^{m-\left|z_{j}\right|}$, $j=1,2, \ldots, p$, for some pairwise distinct $z_{1}, z_{2}, \ldots, z_{p} \in D_{n}$ with $\max _{1 \leq j \leq p}\left|z_{j}\right| \leq$ $k$, then $\Lambda_{p} y[1, k-1] \Lambda_{p}^{m-k}$ is contained in $\bigcup_{j=1}^{p} z_{j} \Lambda_{p}^{m-\left|z_{j}\right|}$, and hence $y[1, k-1] \Lambda_{p}^{m-k}$ is contained in $C_{n+1}$, which contradicts the assumption $y \in D_{n+1}$. Therefore $\Lambda_{p} y \Lambda_{p}^{m-1-k}$ has non-empty intersection with $z \Lambda_{p}^{m-|z|}$ for at most $p-1$ elements $z \in$ $D_{n}$ with $|z| \leq k$. If $\Lambda_{p} y \Lambda_{p}^{m-1-k}$ does have non-empty intersection with $z \Lambda_{p}^{m-|z|}$ for some $z \in D_{n}$ with $|z| \leq k$, then, since $|z| \geq 1$, one sees that $\Lambda_{p} y \Lambda_{p}^{m-1-k} \cap z \Lambda_{p}^{m-|z|}$ is equal to $j y \Lambda_{p}^{m-1-k}$ for some $j \in \Lambda_{p}$. Therefore

$$
\left|\Lambda_{p} y \Lambda_{p}^{m-1-k} \cap\left(\bigcup_{z \in D_{n},|z| \leq k} z \Lambda_{p}^{m-|z|}\right)\right| \leq(p-1)\left|y \Lambda_{p}^{m-1-k}\right|=(p-1) p^{m-1-k},
$$

and hence

$$
\left|\left(\bigcup_{y \in D_{n+1, k}} \Lambda_{p} y \Lambda_{p}^{m-1-k}\right) \cap\left(\bigcup_{z \in D_{n},|z| \leq k} z \Lambda_{p}^{m-|z|}\right)\right| \leq\left|D_{n+1, k}\right|(p-1) p^{m-1-k} .
$$

Note that $\bigcup_{y \in D_{n+1, k}} \Lambda_{p} y \Lambda_{p}^{m-1-k} \subseteq \bigcup_{c \in C_{n+1}} \Lambda_{p} c \subseteq B_{n+1}$. Therefore

$$
\begin{aligned}
\left|\bigcup_{y \in D_{n+1, k}} \Lambda_{p} y \Lambda_{p}^{m-1-k}\right| & =\left|\left(\bigcup_{y \in D_{n+1, k}} \Lambda_{p} y \Lambda_{p}^{m-1-k}\right) \cap B_{n+1}\right| \\
& \underline{\underline{1}} \mid\left(\bigcup_{y \in D_{n+1, k}} \Lambda_{p} y \Lambda_{p}^{m-1-k}\right) \cap\left(A_{n+1} \cup\left(\bigcup_{z \in D_{n}} z \Lambda_{p}^{m-|z|}\right) \mid\right. \\
\leq & \left|\left(\bigcup_{y \in D_{n+1, k}} \Lambda_{p} y \Lambda_{p}^{m-1-k}\right) \cap\left(\bigcup_{z \in D_{n},|z| \leq k} z \Lambda_{p}^{m-|z|}\right)\right| \\
& +\left|\left(\bigcup_{y \in D_{n+1, k}} \Lambda_{p} y \Lambda_{p}^{m-1-|y|}\right) \cap\left(A_{n+1} \cup\left(\bigcup_{z \in D_{n},|z|>k} z \Lambda_{p}^{m-|z|}\right)\right)\right| \\
& \stackrel{\underline{2}}{\leq}\left|D_{n+1, k}\right|(p-1) p^{m-1-k}+\left|A_{n+1} \cup\left(\bigcup_{z \in D_{n},|z|>k} z \Lambda_{p}^{m-|z|}\right)\right| \\
\leq & \left|D_{n+1, k}\right|(p-1) p^{m-1-k}+\ell+\sum_{z \in D_{n},|z|>k} p^{m-|z|} \\
= & \left|D_{n+1, k}\right|(p-1) p^{m-1-k}+\ell+\sum_{j=k+1}^{m-1}\left|D_{n, j}\right| p^{m-j} .
\end{aligned}
$$


Since the sets $\Lambda_{p} y \Lambda_{p}^{m-1-k}$ for $y \in D_{n+1, k}$ are pairwise disjoint, we have

$$
\left|\bigcup_{y \in D_{n+1, k}} \Lambda_{p} y \Lambda_{p}^{m-1-k}\right|=\sum_{y \in D_{n+1, k}}\left|\Lambda_{p} y \Lambda_{p}^{m-1-k}\right|=\left|D_{n+1, k}\right| p^{m-k} .
$$

From (3) and (4) we get

$$
\left|D_{n+1, k}\right| p^{m-1-k} \leq \ell+\sum_{j=k+1}^{m-1}\left|D_{n, j}\right| p^{m-j},
$$

and hence

$$
\left|D_{n+1, k}\right| \leq p^{k+1-m} \ell+\sum_{j=k+1}^{m-1}\left|D_{n, j}\right| p^{|k|+1-j} \leq \ell+\sum_{j=k+1}^{m-1}\left|D_{n, j}\right|,
$$

proving the claim. It follows inductively that $\left|D_{n, m-k}\right| \leq 2^{k-1} \ell$ for all $n \in \mathbb{Z}_{+}$and $1 \leq k \leq m-1$.

We need to show that $B_{n} \neq \Lambda_{p}^{m}$ for all $n \in \mathbb{Z}_{+}$; equivalently, $C_{n} \neq \Lambda_{p}^{m-1}$ for all $n \in \mathbb{Z}_{+}$. In fact, we claim that for every $n \in \mathbb{Z}_{+}$, there are no $d \leq d^{\prime} \leq m-1$ and $y \in \Lambda_{p}^{m-1-d^{\prime}}$ with $y \Lambda_{p}^{d^{\prime}} \subseteq C_{n}$.

We argue by contradiction. So assume that there are $n \in \mathbb{Z}_{+}, d \leq d^{\prime} \leq m-1$ and $y \in \Lambda_{p}^{m-1-d^{\prime}}$ with $y \Lambda_{p}^{d^{\prime}} \subseteq C_{n}$. Let $n_{0}$ be the smallest such $n$, and let $d^{\prime}$ and $y$ witness $n_{0}$. Replacing $y$ by $y w$ for any $w \in \Lambda_{p}^{d^{\prime}-d}$, we may assume that $d^{\prime}=d$.

Since $\frac{\left(n_{0}+1\right) \ell}{p} \geq\left|C_{n_{0}}\right| \geq p^{d}>\frac{(d+p) \ell}{p}$, we have $n_{0} \geq d+p$. Denote $n_{0}-d$ by $n_{1}$. For each $n_{0} \geq n \geq n_{1}$, set $E_{n}$ to be the subset of $C_{n}$ consisting of $c$ satisfying $c\left[1+n_{0}-n, m-1-d+n_{0}-n\right]=y$. The assumption in the above paragraph says that $E_{n_{0}}=y \Lambda_{p}^{d}$ and hence $\left|E_{n_{0}}\right|=p^{d}$. For each $n_{0}>n \geq n_{1}$, we have

$$
\bigcup_{c \in E_{n+1}} \Lambda_{p} c \subseteq \bigcup_{c \in C_{n+1}} \Lambda_{p} c \subseteq B_{n+1}=A_{n+1} \cup\left(\bigcup_{c^{\prime} \in C_{n}} c^{\prime} \Lambda_{p}\right) .
$$

Note that if $\Lambda_{p} c \cap c^{\prime} \Lambda_{p} \neq \emptyset$ for some $c \in E_{n+1}$ and $c^{\prime} \in C_{n}$, then $c^{\prime}$ is in $E_{n}$. Thus

$$
\bigcup_{c \in E_{n+1}} \Lambda_{p} c \subseteq A_{n+1} \cup\left(\bigcup_{c^{\prime} \in E_{n}} c^{\prime} \Lambda_{p}\right)
$$

and hence

$$
p\left|E_{n+1}\right|=\left|\bigcup_{c \in E_{n+1}} \Lambda_{p} c\right| \leq\left|A_{n+1} \cup\left(\bigcup_{c^{\prime} \in E_{n}} c^{\prime} \Lambda_{p}\right)\right| \leq\left|A_{n+1}\right|+p\left|E_{n}\right| \leq \ell+p\left|E_{n}\right| .
$$

It follows inductively that $\left|E_{n}\right| \geq\left|E_{n_{0}}\right|-\frac{\left(n_{0}-n\right) \ell}{p}=p^{d}-\frac{\left(n_{0}-n\right) \ell}{p}$ for all $n_{0} \geq n \geq n_{1}$. In particular, $\left|E_{n_{1}}\right| \geq p^{d}-\frac{d \ell}{p}>\ell$.

Denote $\max \left(0, d+n_{1}-m+1\right)$ by $n_{2}$. For each $n_{1} \geq n \geq n_{2}$ denote by $k_{n}$ the largest number $k$ for which there exists a subset $F$ of $C_{n}$ such that $|F|=k$ and $c\left[1+d+n_{1}-n, m-1\right]$ does not depend on $c \in F$. Taking $F$ to be $E_{n_{1}}$ we see that $k_{n_{1}} \geq\left|E_{n_{1}}\right|>\ell$. We claim that $p k_{n+1} \leq k_{n}+\ell$ for all $n_{1}>n \geq n_{2}$. Take $F \subseteq C_{n+1}$ such that $|F|=k_{n+1}$ and $c\left[d+n_{1}-n, m-1\right]$ does not depend on $c \in F$. Then the set $W:=\bigcup_{c \in F} \Lambda_{p} c$ has $p k_{n+1}$ elements and is contained in $B_{n+1}=A_{n+1} \cup\left(\bigcup_{c^{\prime} \in C_{n}} c^{\prime} \Lambda_{p}\right)$. Set $F^{\prime}=\left\{c^{\prime} \in C_{n}: c^{\prime} \Lambda_{p} \cap W \neq \emptyset\right\}$. Then $c\left[1+d+n_{1}-n, m-1\right]$ does not depend on $c \in F^{\prime}$, and hence $\left|F^{\prime}\right| \leq k_{n}$. Since 
$d+n_{1}-n \leq d+n_{1}-n_{2} \leq m-1$, all the elements in $W$ have the same right end. It follows that for any $c^{\prime} \in F^{\prime}$, one has $\left|W \cap c^{\prime} \Lambda_{p}\right|=1$. Thus

$$
\begin{aligned}
p k_{n+1} & =|W|=\left|W \cap\left(A_{n+1} \cup\left(\bigcup_{c^{\prime} \in C_{n}} c^{\prime} \Lambda_{p}\right)\right)\right|=\left|W \cap\left(A_{n+1} \cup\left(\bigcup_{c^{\prime} \in F^{\prime}} c^{\prime} \Lambda_{p}\right)\right)\right| \\
& \leq\left|A_{n+1}\right|+\sum_{c^{\prime} \in F^{\prime}}\left|W \cap c^{\prime} \Lambda_{p}\right|=\left|A_{n+1}\right|+\left|F^{\prime}\right| \leq \ell+k_{n} .
\end{aligned}
$$

This proves the claim. Inductively, we get $k_{n} \geq p^{n_{1}-n}+\ell$ for all $n_{1} \geq n \geq n_{2}$. In particular, $\left|C_{n_{2}}\right| \geq k_{n_{2}} \geq p^{n_{1}-n_{2}}+\ell$. Since $\left|C_{0}\right| \leq \frac{\ell}{p}$, we have $n_{2}>0$. Thus $n_{2}=d+n_{1}-m+1$, and hence $\left|C_{n_{2}}\right| \geq k_{n_{2}} \geq p^{m-d-1}+\ell$.

Since $n_{2} \leq n_{1}<n_{0}$, according to the choice of $n_{0}, D_{n_{2}, k}$ is empty for all $0 \leq k \leq m-1-d$. Thus

$$
\begin{aligned}
\left|C_{n_{2}}\right| & =\left|\bigcup_{k=0}^{m-1}\left(\bigcup_{y \in D_{n_{2}, k}} y \Lambda_{p}^{m-1-k}\right)\right| \\
& =\left|\bigcup_{k=m-d}^{m-1}\left(\bigcup_{y \in D_{n_{2}, k}} y \Lambda_{p}^{m-1-k}\right)\right| \\
& =\sum_{k=m-d}^{m-1}\left|D_{n_{2}, k}\right| p^{m-1-k} \\
& \leq \sum_{k=m-d}^{m-1} 2^{m-1-k} \ell \cdot p^{m-1-k}=\ell \sum_{j=0}^{d-1}(2 p)^{j}=\frac{(2 p)^{d}-1}{2 p-1} \cdot \ell .
\end{aligned}
$$

This contradicts $\left|C_{n_{2}}\right| \geq p^{m-d-1}+\ell$ once we take $m$ large enough such that $\frac{(2 p)^{d}-1}{2 p-1}$. $\ell<p^{m-1-d}+\ell$. A simple calculation shows that we may take $d=\ell+1$ and $m \geq 4 \ell+2$.

\section{ACKNOWLEDGEMENTS}

Part of this work was carried out during visits of the second author to the first and third authors in the summers of 2008 and 2009. The second author is grateful to them for their warm hospitality. The authors thank the referees for very helpful comments.

\section{REFERENCES}

[1] E. Akin. Recurrence in Topological Dynamical Systems. Furstenberg Families and Ellis Actions. The University Series in Mathematics. Plenum Press, New York, 1997. MR.1467479 (2000c:37014)

[2] V. Bergelson. Sets of recurrence of $\mathbb{Z}^{m}$-actions and properties of sets of differences in $\mathbb{Z}^{m} . J$. London Math. Soc. (2) 31 (1985), 295-304. MR809951 (87a:28025)

[3] V. Bergelson. Minimal idempotents and ergodic Ramsey theory. In: Topics in Dynamics and Ergodic Theory, pp. 8-39, London Math. Soc. Lecture Note Ser., 310, Cambridge Univ. Press, Cambridge, 2003. MR2052273 (2006b:37022)

[4] F. Blanchard. Fully positive topological entropy and topological mixing. In: Symbolic Dynamics and its Applications (New Haven, CT, 1991), pp. 95-105, Contemp. Math. 135, Amer. Math. Soc., Providence, RI, 1992. MR.1185082 (93k:58134)

[5] F. Blanchard. A disjointness theorem involving topological entropy. Bull. Math. Soc. France 121 (1993), 465-478. MR.1254749 (95e:54050) 
[6] F. Blanchard, E. Glasner, and B. Host. A variation on the variational principle and applications to entropy pairs. Ergod. Theor. Dyn. Syst. 17 (1997), 29-43. MR.1440766 (98k:54073)

[7] F. Blanchard, B. Host, A. Maass, S. Martinez, and D. J. Rudolph. Entropy pairs for a measure. Ergod. Theor. Dyn. Syst. 15 (1995), 621-632. MR.1346392 (96m:28024)

[8] F. Blanchard and Y. Lacroix. Zero entropy factors of topological flows. Proc. Amer. Math. Soc. 119 (1993), 985-992. MR:1155593 (93m:54066)

[9] A. Blokh and A. Fieldsteel. Sets that force recurrence. Proc. Amer. Math. Soc. 130 (2002), 3571-3578. MR.1920036(2003e:37017)

[10] I. P. Cornfeld, S. V. Fomin, and Ya. G. Sinal̆. Ergodic Theory. Translated from the Russian by A. B. Sosinskiı̌. Grundlehren der Mathematischen Wissenschaften [Fundamental Principles of Mathematical Sciences], 245. Springer-Verlag, New York, 1982. MR832433 (87f:28019)

[11] P. Dong, S. Shao, and X. Ye. Product recurrent properties, disjointness and weak disjointness. Israel J. of Math. 188 (2012), 463-507.

[12] H. Furstenberg. Disjointness in ergodic theory, minimal sets, and a problem in Diophantine approximation. Math. Systems Theory 1 (1967), 1-49. MR0213508(35:4369)

[13] H. Furstenberg. Recurrence in Ergodic Theory and Combinatorial Number Theory. Princeton University Press, Princeton, N.J., 1981. MR603625 (82j:28010)

[14] E. Glasner. Ergodic Theory via Joinings. Mathematical Surveys and Monographs, 101. Amer. Math. Soc., Providence, RI, 2003. MR1958753 (2004c:37011)

[15] E. Glasner. Classifying dynamical systems by their recurrence properties. Topol. Methods Nonlinear Anal. 24 (2004), 21-40. MR2111980(2006b:37024)

[16] E. Glasner. On tame dynamical systems. Colloq. Math. 105 (2006), no. 2, 283-295. MR2237913 (2007d:37005)

[17] E. Glasner. The structure of tame minimal dynamical systems. Ergod. Theor. Dyn. Syst. $\mathbf{2 7}$ (2007), no. 6, 1819-1837. MR2371597 (2008m:37015)

[18] E. Glasner and B. Weiss. Strictly ergodic, uniform positive entropy models. Bull. Soc. Math. France 122 (1994), 399-412. MR1294463 (95k:28035)

[19] E. Glasner and B. Weiss. Topological entropy of extensions. In: Ergodic Theory and its Connections with Harmonic Analysis (Alexandria, 1993), pp. 299-307, London Math. Soc. Lecture Note Ser., 205, Cambridge Univ. Press, Cambridge, 1995. MR.1325706 (96b:54064)

[20] E. Glasner and B. Weiss. Quasi-factors of zero entropy systems. J. Amer. Math. Soc. 8 (1995), 665-686. MR 1270579 (95i:54048)

[21] E. Glasner and B. Weiss. On the interplay between measurable and topological dynamics. In: Handbook of Dynamical Systems. Vol. 1B, pp. 597-648, Elsevier B. V., Amsterdam, 2006. MR2186250 (2006i:37005)

[22] E. Glasner and X. Ye. Local entropy theory. Ergod. Theor. Dyn. Syst. 29 (2009), 321-356. MR 2486773 (2010k:37023)

[23] W. H. Gottschalk and G. A. Hedlund. Topological Dynamics. Amer. Math. Soc. Colloquium Publications, Vol. 36. Amer. Math. Soc., Providence, R.I., 1955. MR0074810 (17:650e)

[24] P. R. Halmos. Lectures on Ergodic Theory. Chelsea Publishing Co., New York, 1960. MR 0111817(22:2677)

[25] N. Hindman. Finite sums from sequences within cells of a partition of N. J. Combinatorial Theory Ser. A 17 (1974), 1-11. MR0349574 (50:2067)

[26] W. Huang. Tame systems and scrambled pairs under an abelian group action. Ergod. Theor. Dyn. Syst. 26 (2006), no. 5, 1549-1567. MR2266373 (2007j:37012)

[27] W. Huang, H. Li, and X. Ye. Localization and dynamical Ramsey property. In preparation.

[28] W. Huang, K. K. Park, and X. Ye. Topological disjointness from entropy zero systems. Bull. Soc. Math. France 135 (2007), 259-282. MR2430193 (2009g:54084)

[29] W. Huang, S. Shao, and X. Ye. Mixing via sequence entropy. In: Algebraic and Topological Dynamics, pp. 101-122, Contemp. Math., 385, Amer. Math. Soc., Providence, RI, 2005. MR 2180232 (2006h:37008)

[30] W. Huang and X. Ye. Topological complexity, return times and weak disjointness. Ergod. Theor. Dyn. Syst. 24 (2004), 825-846. MR2062921 (2005c:37011)

[31] W. Huang and X. Ye. Dynamical systems disjoint from any minimal system. Trans. Amer. Math. Soc. 357 (2005), 669-694. MR2095626 (2005g:37012)

[32] W. Huang and X. Ye. A local variational relation and applications. Israel J. Math. 151 (2006), 237-279. MR2214126 (2006k:37033) 
[33] M. G. Karpovsky and V. D. Milman. Coordinate density of sets of vectors. Discrete Math. 24 (1978), 177-184. MR522926 (80m:05004)

[34] A. S. Kechris. Classical Descriptive Set Theory. Graduate Texts in Mathematics, 156. Springer-Verlag, New York, 1995. MR1321597(96e:03057)

[35] D. Kerr and H. Li. Independence in topological and $C^{*}$-dynamics. Math. Ann. 338 (2007), 869-926. MR2317754 (2009a:46126)

[36] D. Kerr and H. Li. Combinatorial independence in measurable dynamics. J. Funct. Anal. 256 (2009), 1341-1386. MR2490222 (2010j:37009)

[37] R. Kuang and X. Ye. The return times set and mixing for measure preserving transformations. Discrete Contin. Dyn. Syst. 18 (2007), 817-827. MR2318270(2008d:37006)

[38] R. Kuang and X. Ye. Mixing via families for measure preserving transformations. Colloq. Math. 110 (2008), 151-165. MR2353902 (2008k:37015)

[39] W. Parry. Topics in Ergodic Theory. Reprint of the 1981 original. Cambridge Tracts in Mathematics, 75. Cambridge University Press, Cambridge, 2004. MR:2140546 (2005m:37003)

[40] K. E. Petersen. Disjointness and weak mixing of minimal sets. Proc. Amer. Math. Soc. 24 (1970), 278-280. MR0250283(40:3522)

[41] K. E. Petersen. A topologically strongly mixing symbolic minimal set. Trans. Amer. Math. Soc. 148 (1970), 603-612. MR0259884 (41:4513)

[42] V. A. Rohlin. Lectures on the entropy theory of measure-preserving transformations with invariant measure. Uspehi Mat. Nauk 22 (1967), 3-56. Translated in Russian Math. Surveys (22) (1967), 1-52. MR0217258 (36:349)

[43] V. A. Rohlin and Ja. G. Sinaľ. The structure and properties of invariant measurable partitions. (Russian) Dokl. Akad. Nauk SSSR 141 (1961), 1038-1041. MR0152629 (27:2604)

[44] A. Rosenthal. Strictly ergodic models for noninvertible transformations. Israel J. Math. 64 (1988), 57-72. MR981749 (90a:28026)

[45] H. P. Rosenthal. A characterization of Banach spaces containing $\ell^{1}$. Proc. Nat. Acad. Sci. U.S.A. 71 (1974), 2411-2413. MR0358307(50:10773)

[46] H. P. Rosenthal. Some recent discoveries in the isomorphic theory of Banach spaces. Bull. Amer. Math. Soc. 84 (1978), 803-831. MR499730 (80d:46023)

[47] K. Roth. Sur quelques ensembles d'entiers. C. R. Acad. Sci. Paris 234 (1952), 388-390. MR0046374 (13:724d)

[48] N. Sauer. On the density of families of sets. J. Combinatorial Theory Ser. A 13 (1972), 145-147. MR 0307902 (46:7017)

[49] S. Shelah. A combinatorial problem; stability and order for models and theories in infinitary languages. Pacific J. Math. 41 (1972), 247-261. MR0307903(46:7018)

[50] J. de Vries. Elements of Topological Dynamics. Mathematics and its Applications, 257. Kluwer Academic Publishers Group, Dordrecht, 1993. MR,1249063 (94m:54098)

[51] P. Walters. An Introduction to Ergodic Theory. Graduate Texts in Mathematics, 79. SpringerVerlag, New York-Berlin, 1982. MR648108 (84e:28017)

[52] B. Weiss. A survey of generic dynamics. In: Descriptive Set Theory and Dynamical Systems (Marseille-Luminy, 1996), pp. 273-291, London Math. Soc. Lecture Note Ser., 277, Cambridge Univ. Press, Cambridge, 2000. MR.1774430 (2001j:37008)

[53] X. Ye and R. Zhang. On sensitive sets in topological dynamics. Nonlinearity 21 (2008), 1601-1620. MR2425336 (2009j:37017)

Department of Mathematics, University of Science and Technology of China, Hefei, Anhui 230026, People's Republic of China

E-mail address: wenh@mail.ustc.edu.cn

Department of Mathematics, Chongqing University, Chongqing 401331, People's Republic of China - and - Department of Mathematics, SUNY at Buffalo, Buffalo, New YORK 14260-2900

E-mail address: hfli@math.buffalo.edu

Department of Mathematics, University of Science and Technology of China, Hefei, Anhui 230026, People's Republic of China

E-mail address: yexd@ustc.edu.cn 